\title{
INFINITE PRODUCT FORMULAE FOR GENERATING FUNCTIONS FOR SEQUENCES OF SQUARES
}

\author{
CHRISTIAN KRATTENTHALER ${ }^{\dagger}$, MIRCEA MERCA, AND CRISTIAN-SILVIU RADU $^{\dagger}$
}

\begin{abstract}
We state and prove product formulae for several generating functions for sequences $\left(a_{n}\right)_{n \geq 0}$ that are defined by the property that $P a_{n}+b^{2}$ is a square, where $P$ and $b$ are given integers. In particular, we prove corresponding conjectures of the second author. We show that, by means of the Jacobi triple product identity, all these generating functions can be reduced to a linear combination of theta function products. The proof of our formulae then consists in simplifying these linear combinations of theta products into single products. We do this in two ways: (1) by using modular function theory, and (2) by applying the Weierstraß addition formula for theta products.
\end{abstract}

\section{IntRODUCTION}

Relations between infinite $q$-series and infinite $q$-products have an honorable history starting with Euler and Gauß and first studied systematically by Jacobi. Many identities of the form

$$
\text { infinite } q \text {-series }=\text { infinite } q \text {-product }
$$

arise in number theory, analysis, combinatorics, the theory of integer partitions, representation theory of Lie algebras, vertex operator algebras, knot theory, and statistical mechanics. Playing with series and products, Euler discovered the pentagonal number theorem (cf. e.g. [2, Ex. 2.18]),

$$
\sum_{n=-\infty}^{\infty}(-1)^{n} q^{n(3 n-1) / 2}=(q ; q)_{\infty}
$$

which is the very first theorem that is of this type. Here, and in the following, $q$ is a complex number with $|q|<1$, and the symbol $(a ; q)_{\infty}$ denotes the infinite product

$$
(a ; q)_{\infty}:=\prod_{i=0}^{\infty}\left(1-a q^{i}\right) .
$$

Moreover, we use the short notation

$$
\left(a_{1}, a_{2}, \ldots, a_{m} ; q\right):=\prod_{j=1}^{m}\left(a_{j} ; q\right)_{\infty} .
$$

2010 Mathematics Subject Classification. Primary 11B65; Secondary 05A30, 11F27, 33D05.

Key words and phrases. Jacobi triple product identity, Jacobi theta functions, Weierstraß relation for theta products, modular functions.

†Supported by the Austrian Science Foundation FWF (grant S50-N15) in the framework of the Special Research Program "Algorithmic and Enumerative Combinatorics".

${ }^{1}$ Alternatively, all definitions and identities may be understood in the sense of formal power series in $q$. 
An alternative way to state Euler's identity is as follows:

Let $\left(a_{n}\right)_{n \geq 0}=(0,1,2,5,7,12,15,22,26,35,40, \ldots)$ be the sequence of non-negative integers $m$ such that $24 m+1$ is a square. Then

$$
\sum_{n=0}^{\infty}(-1)^{\lfloor(n+1) / 2\rfloor} q^{a_{n}}=(q ; q)_{\infty} .
$$

In [3], the second author studied — among others — series-product identities of this type and listed several empirically found such formulae, see [3, Ids. 5.1-5.6, Ids. 6.16.14]. A typical example (cf. [3, Id. 6.1] and Theorem 7 below) is the following statement:

Let $\left(a_{n}\right)_{n \geq 0}=(0,4,7,10,21,26,33,59,61,95,108, \ldots)$ be the sequence of non-negative integers $m$ such that $240 m+1$ is a square. Then

$$
\sum_{n=0}^{\infty}(-1)^{\lfloor(n+2) / 4\rfloor} q^{a_{n}}=\frac{\left(q, q^{7}, q^{8} ; q^{8}\right)_{\infty}\left(q^{6}, q^{10} ; q^{16}\right)_{\infty}}{\left(q, q^{4} ; q^{5}\right)_{\infty}} .
$$

This paper started by the observation that, by the use of Jacobi's triple product identity, the proofs of all these conjectured formulae can be reduced to the verification of certain identities between (specialised) Jacobi theta functions. Now, it is a folklore fact that, since these theta functions are modular functions (for certain subgroups of $\mathrm{SL}_{2}(\mathbb{Z})$ ), such identities are routinely verifiable. This is what we did, first. Subsequently, however, we wanted to have a conceptual understanding of the established formulae. Moreover, we were interested in whether there is more than just these, first empirically found, formulae from [3]. Indeed, further computer experiments led us to discover many more such formulae, but still of sporadic nature. Nevertheless, altogether, they helped us to come up with two parametric theorems that subsume several of the earlier empirically found formulae under one roof.

Obviously, parametric theorems cannot be routinely verified anymore. Rather we found that Weierstraß' three-term relation between theta products is the "magic" identity that is behind all of our formulae. More precisely, (aside from four formulae that are direct consequences of the Jacobi triple product identity) for one class of formulae the verification consists in a single application of Weierstraß' relation which reduces the sum of two theta products to a single theta product, while for a second class of formulae the verification requires a double application of Weierstraß' relation - a first application to reduce the sum of four theta products to the sum of two theta products, and then another application to reduce the latter to a single theta product.

Our parametric theorems belong both to the first class. At this point in time, we are not able to offer a conceptual understanding for our proofs for the second class of formulae in the sense that we were not able to embed these into parametric families of formulae. Rather, we performed some computer assisted searches for more formulae in the second class that remained unsuccessful, suggesting that there may not be parametric families in the second class but only these sporadic formulae. 
Our paper is structured as follows. In the next section we collect the empirically found identities from [3]. (We have slightly altered the order in which they are presented in order to provide a more systematic listing.) They become theorems by the proofs in Sections 7 and 9. Section 3 lists the additional identities that we found by our computer experiments subsequent to the publication of [3].

The first step in all our proofs is to apply Jacobi's triple product identity that converts the left-hand sides of our identities into a linear combination of products of theta functions. We recall Jacobi's identity in Section 4, where we also derive two (wellknown) corollaries that we need in some of the proofs.

There follows another section of preparatory character, namely Section 5, in which facts from the theory of modular functions are collected that are relevant in our context. Based on them, we explain in Section 6 how to routinely verify identities between theta products. In the subsequent section, Section 17, we apply this methodology to prove all the theorems from Sections 2 and 3 (with the exception of the theorems that are special cases of parametric families). We provide full details for Theorem 1, while for all other theorems proofs are given in a stenographic fashion since the pattern is always the same.

Section 8 is again preparatory. There, we recall the earlier mentioned Weierstraß' addition formula, and two of its corollaries that we shall use particularly frequently. Then, in Section 9, we present our proofs of all theorems from Sections 2 and 3 (again with the exception of the theorems that are special cases of parametric families) by the use of the Weierstraß relation. Again, we give full details for the proof of Theorem 1, while for all other theorems proofs are presented only in an abridged fashion.

The contents of Section 10 are two parametric families of formulae of the type as in (1.2); one of them consists in Theorem 34 and Corollary 35 (although these contain different statements, their proofs are the same), and the other in Theorem 36 and Corollary 37 (again, these contain different statements, but their proofs are the same). Finally, in Theorem 38 we unify the statements of Theorems 31 and 32 , and we provide a uniform proof.

We close the article by mentioning some consequences and open problems in Section 11.

\section{Generating FunCtions For SEQUences of SQUARES}

Here, we list the empirically found formulae for generating functions for sequences of squares from [3]. They become theorems by the proofs in Sections 7 and 9, respectively.

Theorem 1 (conjectured in [3, Id. 5.1]). Let $\left(a_{n}\right)_{n \geq 0}$ be the sequence of non-negative integers $m$ such that $840 m+361$ is a square. Then

$$
\sum_{n=0}^{\infty}(-1)^{t(n)} q^{a_{n}}=\frac{\left(q, q^{6}, q^{7} ; q^{7}\right)_{\infty}}{\left(q, q^{4} ; q^{5}\right)_{\infty}}
$$

where

$$
t(n)= \begin{cases}0, & \text { if } n \equiv 0,1,3,5,10,12,14,15(\bmod 16) \\ 1, & \text { otherwise }\end{cases}
$$


Theorem 2 (conjectured in [3, Id. 5.2]). Let $\left(a_{n}\right)_{n \geq 0}$ be the sequence of non-negative integers $m$ such that $840 m+529$ is a square. Then

$$
\sum_{n=0}^{\infty}(-1)^{t(n)} q^{a_{n}}=\frac{\left(q, q^{6}, q^{7} ; q^{7}\right)_{\infty}}{\left(q^{2}, q^{3} ; q^{5}\right)_{\infty}}
$$

where

$$
t(n)= \begin{cases}0, & \text { if } n \equiv 0,2,3,6,9,12,13,15(\bmod 16) \\ 1, & \text { otherwise }\end{cases}
$$

Theorem 3 (conjectured in [3, Id. 5.3, corrected]). Let $\left(a_{n}\right)_{n \geq 0}$ be the sequence of non-negative integers $m$ such that $840 m+121$ is a square. Then

$$
\sum_{n=0}^{\infty}(-1)^{\lfloor(n+4) / 8\rfloor} q^{a_{n}}=\frac{\left(q^{2}, q^{5}, q^{7} ; q^{7}\right)_{\infty}}{\left(q, q^{4} ; q^{5}\right)_{\infty}} .
$$

Theorem 4 (conjectured in [3, Id. 5.4]). Let $\left(a_{n}\right)_{n \geq 0}$ be the sequence of non-negative integers $m$ such that $840 m+289$ is a square. Then

$$
\sum_{n=0}^{\infty}(-1)^{t(n)} q^{a_{n}}=\frac{\left(q^{2}, q^{5}, q^{7} ; q^{7}\right)_{\infty}}{\left(q^{2}, q^{3} ; q^{5}\right)_{\infty}}
$$

where

$$
t(n)= \begin{cases}0, & \text { if } n \equiv 0,1,3,5,10,12,14,15(\bmod 16) \\ 1, & \text { otherwise }\end{cases}
$$

Theorem 5 (conjectured in [3, Id. 5.5, corrected]). Let $\left(a_{n}\right)_{n \geq 0}$ be the sequence of non-negative integers $m$ such that $840 m+1$ is a square. Then

$$
\sum_{n=0}^{\infty}(-1)^{\lfloor(n+4) / 8\rfloor} q^{a_{n}}=\frac{\left(q^{3}, q^{4}, q^{7} ; q^{7}\right)_{\infty}}{\left(q, q^{4} ; q^{5}\right)_{\infty}} .
$$

Theorem 6 (conjectured in [3, Id. 5.6]). Let $\left(a_{n}\right)_{n \geq 0}$ be the sequence of non-negative integers $m$ such that $840 m+169$ is a square. Then

$$
\sum_{n=0}^{\infty}(-1)^{t(n)} q^{a_{n}}=\frac{\left(q^{3}, q^{4}, q^{7} ; q^{7}\right)_{\infty}}{\left(q^{2}, q^{3} ; q^{5}\right)_{\infty}}
$$

where

$$
t(n)= \begin{cases}0, & \text { if } n \equiv 0,1,2,4,11,13,14,15(\bmod 16) \\ 1, & \text { otherwise }\end{cases}
$$

Theorem 7 (conjectured in [3, Id. 6.1, corrected]). Let $\left(a_{n}\right)_{n \geq 0}$ be the sequence of non-negative integers $m$ such that $240 m+1$ is a square. Then

$$
\sum_{n=0}^{\infty}(-1)^{\lfloor(n+2) / 4\rfloor} q^{a_{n}}=\frac{\left(q, q^{7}, q^{8} ; q^{8}\right)_{\infty}\left(q^{6}, q^{10} ; q^{16}\right)_{\infty}}{\left(q, q^{4} ; q^{5}\right)_{\infty}} .
$$

Theorem 8 (conjectured in [3, Id. 6.2]). Let $\left(a_{n}\right)_{n \geq 0}$ be the sequence of non-negative integers $m$ such that $240 m+49$ is a square. Then

$$
\sum_{n=0}^{\infty}(-1)^{\lfloor 5 n / 4\rfloor} q^{a_{n}}=\frac{\left(q, q^{7}, q^{8} ; q^{8}\right)_{\infty}\left(q^{6}, q^{10} ; q^{16}\right)_{\infty}}{\left(q^{2}, q^{3} ; q^{5}\right)_{\infty}} .
$$


Theorem 9 (conjectured in [3, Id. 6.5, corrected]). Let $\left(a_{n}\right)_{n \geq 0}$ be the sequence of non-negative integers $m$ such that $240 m+121$ is a square. Then

$$
\sum_{n=0}^{\infty}(-1)^{\lfloor(n+2) / 4\rfloor} q^{a_{n}}=\frac{\left(q^{3}, q^{5}, q^{8} ; q^{8}\right)_{\infty}\left(q^{2}, q^{14} ; q^{16}\right)_{\infty}}{\left(q, q^{4} ; q^{5}\right)_{\infty}} .
$$

Theorem 10 (conjectured in [3, Id. 6.6]). Let $\left(a_{n}\right)_{n \geq 0}$ be the sequence of non-negative integers $m$ such that $240 m+169$ is a square. Then

$$
\sum_{n=0}^{\infty}(-1)^{\lfloor 5 n / 4\rfloor} q^{a_{n}}=\frac{\left(q^{3}, q^{5}, q^{8} ; q^{8}\right)_{\infty}\left(q^{2}, q^{14} ; q^{16}\right)_{\infty}}{\left(q^{2}, q^{3} ; q^{5}\right)_{\infty}} .
$$

Theorem 11 (conjectured in [3, Id. 6.3, corrected]). Let $\left(a_{n}\right)_{n \geq 0}$ be the sequence of non-negative integers $m$ such that $15 m+1$ is a square. Then

$$
\sum_{n=0}^{\infty}(-1)^{\lfloor(n+2) / 4\rfloor} q^{a_{n}}=\frac{\left(q^{2}, q^{6}, q^{8} ; q^{8}\right)_{\infty}\left(q^{4}, q^{12} ; q^{16}\right)_{\infty}}{\left(q, q^{4} ; q^{5}\right)_{\infty}} .
$$

Theorem 12 (conjectured in [3, Id. 6.4, corrected]). Let $\left(a_{n}\right)_{n \geq 0}$ be the sequence of non-negative integers $m$ such that $15 m+4$ is a square. Then

$$
\sum_{n=0}^{\infty}(-1)^{\lfloor(n+2) / 4\rfloor} q^{a_{n}}=\frac{\left(q^{2}, q^{6}, q^{8} ; q^{8}\right)_{\infty}\left(q^{4}, q^{12} ; q^{16}\right)_{\infty}}{\left(q^{2}, q^{3} ; q^{5}\right)_{\infty}} .
$$

Theorem 13 (conjectured in [3, Id. 6.7]). We have

$$
\sum_{n=-\infty}^{\infty} q^{n(5 n+1)}=\frac{\left(q, q^{9}, q^{10} ; q^{10}\right)_{\infty}\left(q^{8}, q^{12} ; q^{20}\right)_{\infty}}{\left(q, q^{4} ; q^{5}\right)_{\infty}}
$$

Theorem 14 (conjectured in [3, Id. 6.8]). We have

$$
\sum_{n=0}^{\infty}\left(q^{n(n+1)}-q^{5 n(n+1)+1}\right)=\frac{\left(q, q^{9}, q^{10} ; q^{10}\right)_{\infty}\left(q^{8}, q^{12} ; q^{20}\right)_{\infty}}{\left(q^{2}, q^{3} ; q^{5}\right)_{\infty}}
$$

Theorem 15 (conjectured in [3, Id. 6.9]). We have

$$
1+\sum_{n=1}^{\infty}\left(q^{n^{2}}+q^{5 n^{2}}\right)=\frac{\left(q^{2}, q^{8}, q^{10} ; q^{10}\right)_{\infty}\left(q^{6}, q^{14} ; q^{20}\right)_{\infty}}{\left(q, q^{4} ; q^{5}\right)_{\infty}} .
$$

Theorem 16 (conjectured in [3, Id. 6.10]). We have

$$
\sum_{n=-\infty}^{\infty} q^{n(5 n+2)}=\frac{\left(q^{2}, q^{8}, q^{10} ; q^{10}\right)_{\infty}\left(q^{6}, q^{14} ; q^{20}\right)_{\infty}}{\left(q^{2}, q^{3} ; q^{5}\right)_{\infty}}
$$

Theorem 17 (conjectured in [3, Id. 6.11]). We have

$$
\sum_{n=0}^{\infty}\left(q^{n(n+1)}+q^{5 n(n+1)+1}\right)=\frac{\left(q^{3}, q^{7}, q^{10} ; q^{10}\right)_{\infty}\left(q^{4}, q^{16} ; q^{20}\right)_{\infty}}{\left(q, q^{4} ; q^{5}\right)_{\infty}}
$$

Theorem 18 (conjectured in [3, Id. 6.12]). We have

$$
\sum_{n=-\infty}^{\infty} q^{n(5 n+3)}=\frac{\left(q^{3}, q^{7}, q^{10} ; q^{10}\right)_{\infty}\left(q^{4}, q^{16} ; q^{20}\right)_{\infty}}{\left(q^{2}, q^{3} ; q^{5}\right)_{\infty}}
$$


Theorem 19 (conjectured in [3, Id. 6.13]). We have

$$
\sum_{n=-\infty}^{\infty} q^{n(5 n+4)}=\frac{\left(q^{4}, q^{6}, q^{10} ; q^{10}\right)_{\infty}\left(q^{2}, q^{18} ; q^{20}\right)_{\infty}}{\left(q, q^{4} ; q^{5}\right)_{\infty}}
$$

Theorem 20 (conjectured in [3, Id. 6.14]). We have

$$
\sum_{n=1}^{\infty}\left(q^{n^{2}-1}-q^{5 n^{2}-1}\right)=\frac{\left(q^{4}, q^{6}, q^{10} ; q^{10}\right)_{\infty}\left(q^{2}, q^{18} ; q^{20}\right)_{\infty}}{\left(q^{2}, q^{3} ; q^{5}\right)_{\infty}}
$$

\section{More Generating FUnCtions FOR SEQUENCES OF SQUARES}

In this section, we collect the additional formulae for generating functions for sequences of squares that we found when we started our work on this kind of identities. Also these become theorems by the proofs in Sections 7 and 9 , respectively.

Theorem 21. Let $\left(a_{n}\right)_{n \geq 0}$ be the sequence of non-negative integers $m$ such that $120 m+$ 49 is a square. Then

$$
\sum_{n=0}^{\infty}(-1)^{\lfloor 5 n / 4\rfloor} q^{a_{n}}=\frac{\left(q, q^{4}, q^{5} ; q^{5}\right)_{\infty}}{\left(q^{2}, q^{3} ; q^{5}\right)_{\infty}}
$$

Theorem 22. Let $\left(a_{n}\right)_{n \geq 0}$ be the sequence of non-negative integers $m$ such that $120 m+1$ is a square. Then

$$
\sum_{n=0}^{\infty}(-1)^{\lfloor(n+2) / 4\rfloor} q^{a_{n}}=\frac{\left(q^{2}, q^{3}, q^{5} ; q^{5}\right)_{\infty}}{\left(q, q^{4} ; q^{5}\right)_{\infty}} .
$$

Theorem 23. Let $\left(a_{n}\right)_{n \geq 0}$ be the sequence of non-negative integers $m$ such that $168 m+$ 121 is a square. Then

$$
\sum_{n=0}^{\infty}(-1)^{\lfloor 5 n / 4\rfloor} q^{a_{n}}=\frac{\left(q, q^{6}, q^{7} ; q^{7}\right)_{\infty}}{\left(q^{3}, q^{4} ; q^{7}\right)_{\infty}}
$$

Theorem 24. Let $\left(a_{n}\right)_{n \geq 0}$ be the sequence of non-negative integers $m$ such that $168 m+1$ is a square. Then

$$
\sum_{n=0}^{\infty}(-1)^{\lfloor(n+2) / 4\rfloor} q^{a_{n}}=\frac{\left(q^{2}, q^{5}, q^{7} ; q^{7}\right)_{\infty}}{\left(q, q^{6} ; q^{7}\right)_{\infty}} .
$$

Theorem 25. Let $\left(a_{n}\right)_{n \geq 0}$ be the sequence of non-negative integers $m$ such that $168 m+$ 25 is a square. Then

$$
\sum_{n=0}^{\infty}(-1)^{\lfloor(n+2) / 4\rfloor} q^{a_{n}}=\frac{\left(q^{3}, q^{4}, q^{7} ; q^{7}\right)_{\infty}}{\left(q^{2}, q^{5} ; q^{7}\right)_{\infty}} .
$$

Theorem 26. Let $\left(a_{n}\right)_{n \geq 0}$ be the sequence of non-negative integers $m$ such that $48 m+1$ is a square. Then

$$
\sum_{n=0}^{\infty}(-1)^{\lfloor(n+2) / 4\rfloor} q^{a_{n}}=\frac{\left(q^{2}, q^{6}, q^{8} ; q^{8}\right)_{\infty}}{\left(q, q^{7} ; q^{8}\right)_{\infty}}
$$


Theorem 27. Let $\left(a_{n}\right)_{n \geq 0}$ be the sequence of non-negative integers $m$ such that $48 m+25$ is a square. Then

$$
\sum_{n=0}^{\infty}(-1)^{\lfloor 5 n / 4\rfloor} q^{a_{n}}=\frac{\left(q^{2}, q^{6}, q^{8} ; q^{8}\right)_{\infty}}{\left(q^{3}, q^{5} ; q^{8}\right)_{\infty}}
$$

Theorem 28. Let $\left(a_{n}\right)_{n \geq 0}$ be the sequence of non-negative integers $m$ such that $21 m+1$ is a square. Then

$$
\sum_{n=0}^{\infty}(-1)^{\lfloor(n+2) / 4\rfloor} q^{a_{n}}=\frac{\left(q, q^{6}, q^{7} ; q^{7}\right)_{\infty}\left(q^{5}, q^{9} ; q^{14}\right)_{\infty}}{\left(q, q^{3} ; q^{4}\right)_{\infty}}
$$

Theorem 29. Let $\left(a_{n}\right)_{n \geq 0}$ be the sequence of non-negative integers $m$ such that $21 m+4$ is a square. Then

$$
\sum_{n=0}^{\infty}(-1)^{\lfloor(n+2) / 4\rfloor} q^{a_{n}}=\frac{\left(q^{2}, q^{5}, q^{7} ; q^{7}\right)_{\infty}\left(q^{3}, q^{11} ; q^{14}\right)_{\infty}}{\left(q, q^{3} ; q^{4}\right)_{\infty}} .
$$

Theorem 30. Let $\left(a_{n}\right)_{n \geq 0}$ be the sequence of non-negative integers $m$ such that $21 m+16$ is a square. Then

$$
\sum_{n=0}^{\infty}(-1)^{\lfloor 5 n / 4\rfloor} q^{a_{n}}=\frac{\left(q^{3}, q^{4}, q^{7} ; q^{7}\right)_{\infty}\left(q, q^{13} ; q^{14}\right)_{\infty}}{\left(q, q^{3} ; q^{4}\right)_{\infty}} .
$$

Theorem 31. Let $\left(a_{n}\right)_{n \geq 0}$ be the sequence of non-negative integers $m$ such that $16 m+1$ is a square. Then

$$
\sum_{n=0}^{\infty} q^{a_{n}}=\frac{\left(q, q^{7}, q^{8} ; q^{8}\right)_{\infty}\left(q^{6}, q^{10} ; q^{16}\right)_{\infty}}{\left(q, q^{3} ; q^{4}\right)_{\infty}}
$$

Theorem 32. Let $\left(a_{n}\right)_{n \geq 0}$ be the sequence of non-negative integers $m$ such that $16 m+9$ is a square. Then

$$
\sum_{n=0}^{\infty} q^{a_{n}}=\frac{\left(q^{3}, q^{5}, q^{8} ; q^{8}\right)_{\infty}\left(q^{2}, q^{14} ; q^{16}\right)_{\infty}}{\left(q, q^{3} ; q^{4}\right)_{\infty}}
$$

\section{The JACOBI TRIPle PRODUCT IDENTITy AND TWO OF ITS CONSEQUENCES}

The purpose of this section is, first of all, to state the Jacobi triple product identity which is ubiquitously used in the proofs of our theorems, and, second, to make two corollaries explicit that we need in the proofs of four of our theorems.

The Jacobi triple product identity says that (cf. [2, Eq. (1.6.1)])

$$
\sum_{n=-\infty}^{\infty}(-1)^{n} q^{\left(\begin{array}{c}
n \\
2
\end{array}\right)} z^{n}=(q, z, q / z ; q)_{\infty}
$$

Letting $q \rightarrow q^{2}$ and setting $z=-q$ in (4.1), we obtain

$$
\sum_{n=-\infty}^{\infty} q^{n^{2}}=\left(q^{2},-q,-q ; q^{2}\right)_{\infty}
$$

Since we have

$$
\sum_{n=1}^{\infty} q^{n^{2}}=\sum_{n=-\infty}^{-1} q^{n^{2}}
$$


the previous identity implies

$$
\sum_{n=1}^{\infty} q^{n^{2}}=\frac{1}{2}\left(\left(q^{2},-q,-q ; q^{2}\right)_{\infty}-1\right)
$$

We shall need this identity in the proofs of Theorems 15 and 20 .

On the other hand, letting $q \rightarrow q^{2}$ and setting $z=-q^{2}$ in (4.1), we obtain

$$
\sum_{n=-\infty}^{\infty} q^{n^{2}+n}=2\left(q^{2},-q^{2},-q^{2} ; q^{2}\right)_{\infty}
$$

Since we have

$$
\sum_{n=0}^{\infty} q^{n^{2}+n}=\sum_{n=-\infty}^{-1} q^{n^{2}+n}
$$

the previous identity implies

$$
\sum_{n=0}^{\infty} q^{n^{2}+n}=\left(q^{2},-q^{2},-q^{2} ; q^{2}\right)_{\infty}
$$

This identity will be used in the proofs of Theorems 14 and 17.

\section{BACKGROUND ON MODULAR FUNCTIONS}

In this section, we give a brief introduction to modular functions, tailored to our purposes. Let $\mathbb{H}:=\{x \in \mathbb{C}: \operatorname{Im}(x)>0\}$ denote the upper half plane. Roughly speaking, modular functions are (certain) meromorphic functions on $\mathbb{H}$ that are invariant under the action of a subgroup $\Gamma$ of $\mathrm{SL}_{2}(\mathbb{Z})$. In our setting, $\Gamma=\Gamma_{1}(N)$ and $N>3$, where

$$
\Gamma_{1}(N):=\left\{\left(\begin{array}{ll}
a & b \\
c & d
\end{array}\right) \in \mathrm{SL}_{2}(\mathbb{Z}): a, d \equiv 1(\bmod N) \text { and } c \equiv 0(\bmod N)\right\} .
$$

The crucial fact on which we base the "methodology" explained in the next section, is the following proposition (cf. [4, Prop. 4.12]).

Proposition 33. Let $f$ be a non-constant meromorphic function on a compact Riemann surface $X$. Then

$$
\sum_{p \in X} \operatorname{ord}_{p}(f)=0
$$

where $\operatorname{ord}_{p}(f)$ is the order of the Laurent series expansion of $f$ about the point $p$.

For $\gamma=\left(\begin{array}{ll}a & b \\ c & d\end{array}\right)$ and $\tau \in \mathbb{H}$ we define $\gamma \tau:=\frac{a \tau+b}{c \tau+d}$. For a subgroup $\Gamma$ of $\operatorname{SL}_{2}(\mathbb{Z})$, we let $A(\Gamma):=\{f$ meromorphic on $\mathbb{H}: f(\gamma \tau)=f(\tau)$ for all $\gamma \in \Gamma$ and $\tau \in \mathbb{H}\}$.

A function $f \in A\left(\Gamma_{1}(N)\right)$ can be viewed as a meromorphic function $\tilde{f}$ on the Riemann surface $\mathbb{H} / \Gamma_{1}(N):=\{[\tau]: \tau \in \mathbb{H}\}$. Here, $[\tau]:=\left\{\gamma \tau: \gamma \in \Gamma_{1}(N)\right\}$ denotes the orbit of $\tau \in \mathbb{H}$ under the action of the group $\Gamma_{1}(N)$, and $\tilde{f}([\tau]):=f(\tau)$. One equips $\mathbb{H} / \Gamma_{1}(N)$ with the quotient topology so that it is a topological space. To make $\mathbb{H} / \Gamma_{1}(N)$ into a Riemann surface, we follow the recipe in [5, Sec. 1.8] (cf. also [6]). Then $\tilde{f}$ becomes a meromorphic function on $\mathbb{H} / \Gamma_{1}(N)$. However, as we shall explain in Section 6, we want to use Proposition 33, which is an assertion on meromorphic functions on a compact Riemann surface. In other words, we would need $\mathbb{H} / \Gamma_{1}(N)$ to be compact, which it is 
not. So we need to add some points in order to make it compact. In order to achieve this, we define $\mathbb{H}^{*}:=\mathbb{H} \cup \mathbb{Q} \cup\{i \infty\}$. We extend the action of $\gamma=\left(\begin{array}{ll}a & b \\ c & d\end{array}\right)$ to $\mathbb{H}^{*}$ as follows. For $\frac{s}{t} \in \mathbb{Q}$, we let $\gamma \frac{s}{t}:=\frac{a(s / t)+b}{c(s / t)+d}$ if $c(s / t)+d \neq 0$ and $\gamma \frac{s}{t}:=i \infty$ otherwise. Moreover, we let $\gamma(i \infty)=\frac{a}{c}$ if $c \neq 0$ and $\gamma(i \infty):=i \infty$ otherwise. Then $\mathbb{H}^{*} / \Gamma_{1}(N):=\left\{[\tau]: \tau \in \mathbb{H}^{*}\right\}$ is a compact topological space when equipped with the quotient topology. More precisely, the topology of $\mathbb{H}^{*}$ is generated by the topology of $\mathbb{H}$ (which inherits the standard topology in $\mathbb{C}$ ) and the sets $U_{M}:=\{x \in \mathbb{H}: \operatorname{Im}(x)>M\} \cup\{i \infty\}$, and the sets $\gamma U_{M}$ for all $\gamma \in \mathrm{SL}_{2}(\mathbb{Z})$, where $M$ is a positive real number.

In particular $X_{1}(N):=\mathbb{H}^{*} / \Gamma_{1}(N)$ is made into a Riemann surface following the recipe given in [5, Sec. 1.8] (cf. also [6]). Again, this creates a problem: for a function $f \in A\left(\Gamma_{1}(N)\right.$, the corresponding function $\tilde{f}$ on the quotient space is not necessarily meromorphic on $X_{1}(N)$. The problematic points are the cusps $\{[s / t]: s / t \in \mathbb{Q} \cup\{i \infty\}\}$. Given a function $f \in A\left(\Gamma_{1}(N)\right)$, in order for $\tilde{f}$ to be meromorphic on $X_{1}(N)$, we need that, for each reduced fraction $\frac{a}{c} \in \mathbb{Q}, f$ can be expressed as a Laurent series with finite principal part in powers of $e^{\frac{2 \pi i \gamma-1}{h_{c}}}$. Here, $h_{c}:=N / \operatorname{gcd}(c, N)$ is called the width of the cusps $a / c$. When these additional conditions are satisfied we say that $f$ is a modular function for the group $\Gamma_{1}(N)$, and we write $f \in M\left(\Gamma_{1}(N)\right)$. In particular, $f \in M\left(\Gamma_{1}(N)\right)$ implies that $\tilde{f}$ is meromorphic on $X_{1}(N)$. Furthermore, $\operatorname{ord}_{[a / c]} \tilde{f}$ equals the order of the Laurent series of $f$ in powers of $e^{\frac{2 \pi i \gamma-1}{h_{c}}}$. For an arbitrary function $f \in M\left(\Gamma_{1}(N)\right)$, the order of $\tilde{f}$ at a point $\left[\tau_{0}\right]$ for $\tau_{0} \in \mathbb{H}$ is $t$, where $t$ is the order of $f$ when expanded in powers of $\tau-\tau_{0}$.

\section{How TO "MECHANICALLY" PROVE THETA FUNCTION IDENTITIES}

Here we outline the proof strategy for identities equating sums of theta products that we are going to apply in the proofs of our theorems from Sections 2 and 3 given in the next section.

Let us fix a positive modulus $N>3$. For $g \in\{0, \ldots, N-1\}$ define

$$
E_{g}=E_{g}(q ; N):=q^{N B_{2}(g / N) / 2}\left(q^{g}, q^{N-g} ; q^{N}\right)_{\infty},
$$

where $B_{2}(x)=x^{2}-x+\frac{1}{6}$ is the second Bernoulli polynomial. The function $E_{g}$ is essentially a (specialised) Jacobi theta function, "essentially" referring to the missing factor $\left(q^{N} ; q^{N}\right)_{\infty}$. In the following we explain how identities of the form

$$
\sum_{j=1}^{r} c_{j} \prod_{g} E_{g}^{a_{g}^{(j)}}=0
$$

where the $c_{j}$ 's are complex numbers and the $a_{g}^{(j)}$ 's are integers, can be routinely verified if each summand in the sum above is a modular function for the group $\Gamma_{1}(N)$. It should be noted that the left-hand side of (6.2) is a linear combination of theta products.

Now, to verify the identity (6.2), the following steps have to be performed. For convenience, in the following we write LHS for the left-hand side of (6.2). 
SteP 1. According to [8, Prop. 3], $\prod_{g} E_{g}^{a_{g}}(q ; N)$ is a modular function in $\tau$ for the group $\Gamma_{1}(N)$, where $q=e^{2 \pi i \tau}$, that is, $\prod_{g} E_{g}^{a_{g}}$ is an element of $M\left(\Gamma_{1}(N)\right)$ if

$$
\sum_{g} a_{g} \equiv 0 \quad(\bmod 12) \quad \text { and } \quad \sum_{g} g^{2} a_{g} \equiv 0 \quad(\bmod y(N))
$$

where $y(N)=2 N$ if $N$ is even, and $y(N)=N$ if $N$ is odd. We use this criterion in LHS for each summand in order to check that each summand is a modular function for $\Gamma_{1}(N)$.

STEP 2. Representatives of the cusps for the group $\Gamma_{1}(N)$ are computed. (There are only finitely many.) The computer algebra programme Magma provides an implementation in form of the function Cusps (Gamma1 $(N))$.

STEP 3. For each (representative of a) cusp - except $i \infty, c$ say, and $j=1,2, \ldots, r$, the order of the function $\prod_{g} E_{g}^{a_{g}^{(j)}}$ at $c$ has to be computed. According to [8, Prop. 4], the order $\operatorname{ord}\left(E_{g} ; c, N\right)$ of the function $E_{g}$ at the cusp $c$ of the group $\Gamma_{1}(N)$ is given by

$$
\operatorname{ord}\left(E_{g} ; c, N\right)=\frac{1}{2} \operatorname{gcd}\left(D_{c}, N\right) B_{2}\left(\left\{N_{c} g / \operatorname{gcd}\left(D_{c}, N\right)\right\}\right),
$$

where $D_{c}$ is the denominator of $c$ and $N_{c}$ is the numerator of $c$, while $\{\alpha\}$ denotes the fractional part of the rational number $\alpha$. The order of $\prod_{g} E_{g}^{a_{g}^{(j)}}$ at $c$ then is

$$
\sum_{g} a_{g}^{(j)} \operatorname{ord}\left(E_{g} ; c, N\right)
$$

STEP 4. We obtain a lower bound on the order of LHS at $c$ by taking the minimum of the orders of the individual summands of the sum in LHS.

SteP 5. By Proposition 33 , if $L H S$ is not identically zero, then the sum of all the orders equals zero. Hence, (again assuming that LHS is not identically zero) for the function $\widetilde{L H S}$ on the compact Riemann surface $X_{1}(N)$, we have

$$
0=\operatorname{ord}_{[i \infty]}(\widetilde{L H S})+\sum_{[c], c \text { a cusp, }[c] \neq[i \infty]} \operatorname{ord}_{[c]}(\widetilde{L H S})+\sum_{[p], p \text { not a cusp }} \operatorname{ord}_{[p]}(\widetilde{L H S}) .
$$

If we sum all the lower bounds that we found in Step 4 over all the cusps different from $i \infty$, then we obtain a lower bound, $-U$ say, on the first sum in the above expression. Furthermore, from the definition of the function $E_{g}$ it is obvious that it cannot have a singularity at a point $p \in \mathbb{H}$, and thus the order of $E_{g}$ at $p$ is non-negative. This implies directly that the orders ord $\operatorname{orp}_{[p]}(\widetilde{L H S})$ are non-negative, yielding the lower bound 0 on the second sum. Everything combined, we see that ord ${ }_{[i \infty]}(\widetilde{L H S}) \leq U$.

STEP 6. We now verify by direct computation that LHS has the power series expansion $0+0 q+\cdots+0 q^{U}+\cdots$. This says that $\operatorname{ord}_{[i \infty]}(\widetilde{L H S})>U$ (the reader should recall that, under the relation $q=e^{2 \pi i \tau}$, the point $\tau=i \infty$ corresponds to $q=0$ ), a contradiction to our finding in Step 5 under the assumption that $L H S$ is not identically zero. Consequently, LHS must be the zero function. 


\section{7. "MECHANICAL" PROOFS}

This section is devoted to the presentation of the proofs of the theorems in Sections 2 and 3 that are based on the procedure outlined in the previous section. We provide full details for the proof of Theorem 1, while we remain brief for the proofs of the other theorems, all of them being completely analogous. For the theorems which are specialisations of the parametric theorems in Section 10, we refer to the proofs given there.

Proof of Theorem 1. STEP 0. We write the sum on the left-hand side of (2.1) explicitly, and then apply the Jacobi triple product identity to obtain an expression that is a linear combination of products of theta functions.

In order to accomplish this, we first observe that squares that are congruent to 361 modulo 840 are of the form $S^{2}$, where $S \equiv 19,61,79,89,121,131,149,191(\bmod 210)$. Consequently, taking the definition of $t(n)$ into account, we have

$$
\begin{gathered}
\sum_{n=0}^{\infty}(-1)^{t(n)} q^{a_{n}}=\sum_{k=0}^{\infty}(-1)^{k} q^{\frac{1}{840}\left((210 k+19)^{2}-361\right)}+\sum_{k=0}^{\infty}(-1)^{k} q^{\frac{1}{840}\left((210 k+61)^{2}-361\right)} \\
-\sum_{k=0}^{\infty}(-1)^{k} q^{\frac{1}{840}\left((210 k+79)^{2}-361\right)}+\sum_{k=0}^{\infty}(-1)^{k} q^{\frac{1}{840}\left((210 k+89)^{2}-361\right)} \\
-\sum_{k=0}^{\infty}(-1)^{k} q^{\frac{1}{840}\left((210 k+121)^{2}-361\right)}+\sum_{k=0}^{\infty}(-1)^{k} q^{\frac{1}{840}\left((210 k+131)^{2}-361\right)} \\
\quad-\sum_{k=0}^{\infty}(-1)^{k} q^{\frac{1}{840}\left((210 k+149)^{2}-361\right)}-\sum_{k=0}^{\infty}(-1)^{k} q^{\frac{1}{840}\left((210 k+191)^{2}-361\right)} \\
=\sum_{k=0}^{\infty}(-1)^{k} q^{\frac{105 k^{2}}{2}+\frac{19 k}{2}}+\sum_{k=0}^{\infty}(-1)^{k} q^{\frac{105 k^{2}}{2}+\frac{61 k}{2}+4}-\sum_{k=0}^{\infty}(-1)^{k} q^{\frac{105 k^{2}}{2}+\frac{79 k}{2}+7} \\
+\sum_{k=0}^{\infty}(-1)^{k} q^{\frac{105 k^{2}}{2}+\frac{89 k}{2}+9}-\sum_{k=0}^{\infty}(-1)^{k} q^{\frac{105 k^{2}}{2}+\frac{121 k}{2}+17}+\sum_{k=0}^{\infty}(-1)^{k} q^{\frac{105 k^{2}}{2}+\frac{131 k}{2}+20} \\
-\sum_{k=0}^{\infty}(-1)^{k} q^{\frac{105 k^{2}}{2}+\frac{149 k}{2}+26}-\sum_{k=0}^{\infty}(-1)^{k} q^{\frac{105 k^{2}}{2}+\frac{191 k}{2}+43} .
\end{gathered}
$$

By performing the replacement $k \rightarrow-k-1$, we see that the last sum on the right-hand side of (7.1) can be rewritten as

$$
\sum_{k=0}^{\infty}(-1)^{k} q^{\frac{105 k^{2}}{2}+\frac{191 k}{2}+43}=\sum_{k=-\infty}^{-1}(-1)^{k+1} q^{\frac{105 k^{2}}{2}+\frac{19 k}{2}}
$$

Thus, it can be combined with the first sum on the right-hand side of (7.1). This is similar for the other sums. As a result, they can be paired so that one obtains four 
sums over all integers $k$ :

$$
\begin{aligned}
\sum_{n=0}^{\infty}(-1)^{t(n)} q^{a_{n}}= & \sum_{k=-\infty}^{\infty}(-1)^{k} q^{\frac{105 k^{2}}{2}}+\frac{19 k}{2} \\
& +\sum_{k=-\infty}^{\infty}(-1)^{k} q^{\frac{105 k^{2}}{2}+\frac{61 k}{2}+4} \\
& -\sum_{k=-\infty}^{\infty}(-1)^{k} q^{\frac{105 k^{2}}{2}+\frac{79 k}{2}+7}+\sum_{k=-\infty}^{\infty}(-1)^{k} q^{\frac{105 k^{2}}{2}+\frac{89 k}{2}+9} .
\end{aligned}
$$

Now, as announced, to each of these sums we apply the Jacobi triple product identity (4.1) to get

$$
\begin{aligned}
\sum_{n=0}^{\infty}(-1)^{t(n)} q^{a_{n}}=\left(q^{105}, q^{62}, q^{43} ; q^{105}\right)_{\infty}+q^{4}\left(q^{105}, q^{83}, q^{22} ; q^{105}\right)_{\infty} & \\
& -q^{7}\left(q^{105}, q^{92}, q^{13} ; q^{105}\right)_{\infty}+q^{9}\left(q^{105}, q^{97}, q^{8} ; q^{105}\right)_{\infty} .
\end{aligned}
$$

Thus, we have to prove the identity

$$
\begin{aligned}
0=\left(q^{105}, q^{62}, q^{43}\right. & \left.; q^{105}\right)_{\infty}+q^{4}\left(q^{105}, q^{83}, q^{22} ; q^{105}\right)_{\infty} \\
& -q^{7}\left(q^{105}, q^{92}, q^{13} ; q^{105}\right)_{\infty}+q^{9}\left(q^{105}, q^{97}, q^{8} ; q^{105}\right)_{\infty}-\frac{\left(q, q^{6}, q^{7} ; q^{7}\right)_{\infty}}{\left(q, q^{4} ; q^{5}\right)_{\infty}} .
\end{aligned}
$$

We divide both sides of the identity by the first term on the right-hand side and obtain

$$
\begin{aligned}
0=1+q^{4} \frac{\left(q^{105}, q^{83}, q^{22} ; q^{105}\right)_{\infty}}{\left(q^{105}, q^{62}, q^{43} ; q^{105}\right)_{\infty}}-q^{7} \frac{\left(q^{105}, q^{92}, q^{13} ; q^{105}\right)_{\infty}}{\left(q^{105}, q^{62}, q^{43} ; q^{105}\right)_{\infty}} \\
+q^{9} \frac{\left(q^{105}, q^{97}, q^{8} ; q^{105}\right)_{\infty}}{\left(q^{105}, q^{62}, q^{43} ; q^{105}\right)_{\infty}}-\frac{\left(q, q^{6}, q^{7} ; q^{7}\right)_{\infty}}{\left(q, q^{4} ; q^{5}\right)_{\infty}\left(q^{105}, q^{62}, q^{43} ; q^{105}\right)_{\infty}} .
\end{aligned}
$$

Now we fix $N:=105$. With the notation (6.1), our identity can be written as

$$
0=1+\frac{E_{22}}{E_{43}}-\frac{E_{13}}{E_{43}}+\frac{E_{8}}{E_{43}}-\frac{E_{7} E_{8} E_{13} E_{15} E_{20} E_{22} E_{27} E_{28} E_{35} E_{42} E_{48} E_{50}}{E_{4} E_{9} E_{11} E_{16} E_{19} E_{24} E_{26} E_{31} E_{39} E_{44} E_{46} E_{51}} .
$$

In order to rewrite the last term we used that

$$
\left(q^{7} ; q^{7}\right)=\left(q^{7}, q^{14}, q^{21}, \ldots, q^{105} ; q^{105}\right)
$$

and similar "blow-ups" for other terms.

STEP 1. We use the criterion (6.3) to see that all summands on the right-hand side of (7.3) are modular functions for $\Gamma_{1}(105)$. Indeed, for $N=105$ and $\frac{E_{22}}{E_{43}}$, we have $1-1 \equiv 0(\bmod 12)$ and $22^{2}-43^{2} \equiv 0(\bmod 105)$. This shows that $\frac{E_{22}}{E_{43}}$ is a modular function for the group $\Gamma_{1}(105)$. Similarly, we observe that $\frac{E_{13}}{E_{43}}, \frac{E_{8}}{E_{43}}$ and $\frac{E_{7} E_{8} E_{13} E_{15} E_{20} E_{22} E_{27} E_{28} E_{35} E_{42} E_{48} E_{50}}{E_{4} E_{9} E_{11} E_{16} E_{19} E_{24} E_{26} E_{31} E_{39} E_{44} E_{46} E_{51}}$ are modular functions for the group $\Gamma_{1}(105)$. Consequently,

$$
f:=1+\frac{E_{22}}{E_{43}}-\frac{E_{13}}{E_{43}}+\frac{E_{8}}{E_{43}}-\frac{E_{7} E_{8} E_{13} E_{15} E_{20} E_{22} E_{27} E_{28} E_{35} E_{42} E_{48} E_{50}}{E_{4} E_{9} E_{11} E_{16} E_{19} E_{24} E_{26} E_{31} E_{39} E_{44} E_{46} E_{51}}
$$

is a modular function for the group $\Gamma_{1}(105)$.

STEP 2. We use the computer algebra Magma to compute representatives of the cusps for the group $\Gamma_{1}(105)$. This is done by using the command Cusps (Gamma1(105)). The output is 
$[0 o, 0,1 / 13,1 / 12,2 / 23,1 / 11,3 / 32,2 / 21,1 / 10,3 / 29,5 / 48,2 / 19,3 / 28,4 / 37,1 / 9, \backslash$ $5 / 44,4 / 35,3 / 26,8 / 69,5 / 43,2 / 17,3 / 25,4 / 33,1 / 8,6 / 47,5 / 39,9 / 70,4 / 31,11 / 84, \backslash$ $13 / 99,5 / 38,7 / 53,2 / 15,13 / 96,8 / 59,3 / 22,7 / 51,1 / 7,5 / 34,4 / 27,29 / 195,18 / 121, \backslash$ $3 / 20,48 / 319,79 / 525,5 / 33,16 / 105,7 / 45,18 / 115,8 / 51,4 / 25,17 / 105,1 / 6,6 / 35, \backslash$ $11 / 63,18 / 103,7 / 40,8 / 45,23 / 129,5 / 28,7 / 39,9 / 50,11 / 60,9 / 49,12 / 65,5 / 27, \backslash$ $19 / 102,30 / 161,13 / 69,17 / 90,4 / 21,1 / 5,109 / 525,27 / 130,19 / 91,23 / 110,22 / 105, \backslash$ $47 / 222,18 / 85,33 / 155,3 / 14,14 / 65,68 / 315,13 / 60,41 / 189,64 / 295,29 / 133, \backslash$ $19 / 87,26 / 119,23 / 105,9 / 41,20 / 91,11 / 50,11 / 49,9 / 40,71 / 315,23 / 102,30 / 133, \backslash$ $17 / 75,27 / 119,8 / 35,13 / 56,44 / 189,7 / 30,13 / 55,5 / 21,6 / 25,9 / 35,11 / 42,59 / 225, \backslash$ $37 / 140,23 / 87,53 / 200,13 / 49,4 / 15,62 / 231,51 / 190,47 / 175,32 / 119,368 / 1365, \backslash$ $17 / 63,13 / 48,29 / 105,31 / 112,46 / 165,41 / 147,59 / 210,69 / 245,2 / 7,13 / 45, \backslash$ $71 / 245,7 / 24,92 / 315,45 / 154,43 / 147,31 / 105,34 / 115,29 / 98,52 / 175, \backslash$ $25 / 84,94 / 315,19 / 63,32 / 105,13 / 42,24 / 77,11 / 35,16 / 45,113 / 315,48 / 133, \backslash$ $38 / 105,23 / 63,11 / 30,31 / 84,13 / 35,37 / 98,8 / 21,67 / 175,523 / 1365,29 / 75,1$ $64 / 165,41 / 105,124 / 315,2 / 5,43 / 105,41 / 100,26 / 63,31 / 75,44 / 105,103 / 245, \backslash$ $47 / 105,16 / 35,7 / 15,8 / 15,19 / 35,39 / 70,137 / 245,47 / 84,17 / 30,4 / 7,97 / 168, \backslash$ $41 / 70,37 / 63,13 / 21,152 / 245,87 / 140,22 / 35,19 / 30,24 / 35,46 / 63,11 / 15, \backslash$ $23 / 30,27 / 35]\}$.

STEP 3. We now compute the order of each summand of LHS at each cusp except $i \infty$. To do this, we have to use (6.4). Assume for example that we want to compute the order at the cusp $27 / 35$ of the function $\frac{E_{22}}{E_{43}}$. It is convenient to implement the following two functions in Maple:

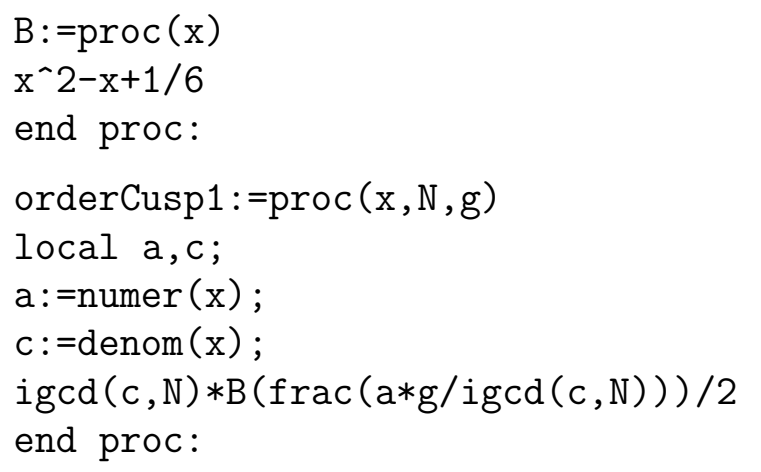

Now, in order to achieve our task we enter in Maple:

orderCusp1 $(27 / 35,105,22)$-orderCusp1 $(27 / 35,105,43)$;

2

The output means that $\frac{E_{22}}{E_{43}}$ has a zero of order 2 at the cusp $\frac{27}{35}$.

STEP 4. We want to compute a lower bound on the order of $f$ at the cusp $27 / 35$. In order to do this, we do the above computation for each term in $f$ and take the minimum of all orders. This computation can be simplified by the function

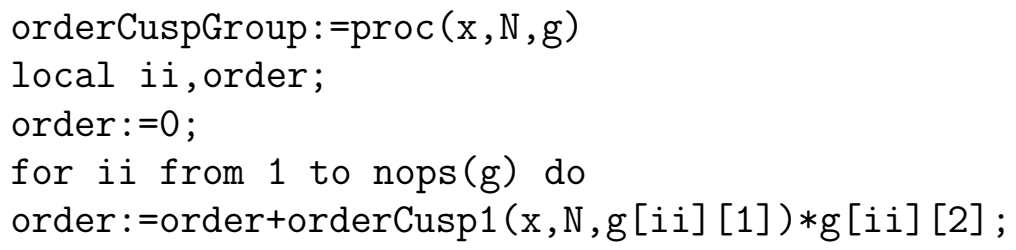


od;

order

end proc:

This function takes as input the cusp representative $x$, the $N$ - which in our case is $105-$, and the index $g$ from $E_{g}$. The product $\frac{E_{22}}{E_{43}}$ is expressed as

$\mathrm{f} 1:=[[22,1],[43,-1]]$;

Then we can compute the order of $\frac{E_{22}}{E_{43}}$ at the cusp $27 / 35$ by typing orderCuspGroup $(27 / 35,105, \mathrm{f} 1)$;

2

We define the other terms by

$\mathrm{f} 2:=[[8,1],[43,-1]]$;

$\mathrm{f} 3:=[[13,1],[43,-1]]$;

$\mathrm{f} 4:=[[7,1],[8,1],[13,1],[15,1],[20,1],[22,1],[27,1],[28,1]$,

$[35,1],[42,1],[48,1],[50,1],[4,-1],[9,-1],[11,-1],[16,-1]$, $[19,-1],[24,-1],[26,-1],[31,-1],[39,-1],[44,-1],[46,-1],[51,-1]]$;

Now we can get a lower bound on the order of $f$ at the cusp $27 / 35$ by writing

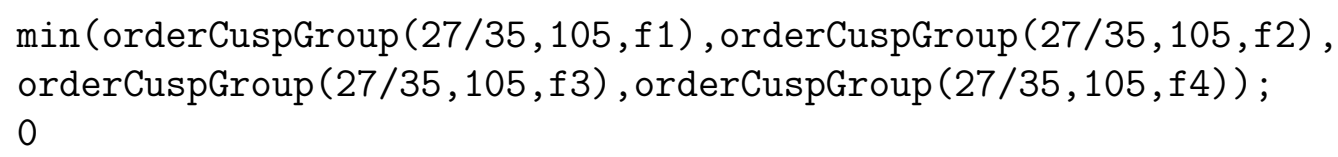

Hence, our lower bound is 0 .

STEP 5. We sum up the lower bounds on the orders at all cusps except $i \infty$. Thus, we obtain an upper bound on the order of $\tilde{f}$ at $[i \infty]$. This is done as follows:

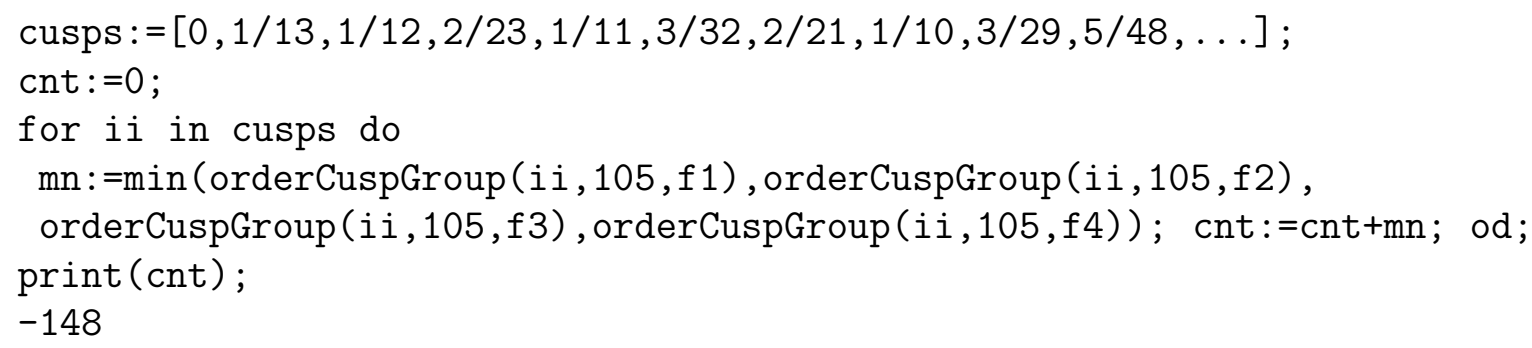

This means that the order of $\tilde{f}$ at $i \infty$ is at most 148, under the assumption that $f$ is not identically zero.

STEP 6. It is routine to verify that $f=0+0 q+\cdots+0 q^{148}+\cdots$. This implies that $\operatorname{ord}_{[i \infty]}(\tilde{f})>148$. Hence, $f$ must be the zero function.

Proof of Theorem 2. It is elementary to see that squares that are congruent to 529 modulo 840 are of the form $S^{2}$, where $S \equiv 23,37,47,103,107,163,173,187(\bmod 210)$. If we now do a computation analogous to the one in the proof of Theorem 1, we obtain

$$
\begin{aligned}
\sum_{n=0}^{\infty}(-1)^{t(n)} q^{a_{n}}=\left(q^{105}, q^{64}, q^{41}\right. & \left.; q^{105}\right)_{\infty}-q\left(q^{105}, q^{71}, q^{34} ; q^{105}\right)_{\infty} \\
& +q^{2}\left(q^{105}, q^{76}, q^{29} ; q^{105}\right)_{\infty}+q^{12}\left(q^{105}, q^{104}, q ; q^{105}\right)_{\infty}
\end{aligned}
$$


Continuing as in the previous proof, we observe that the assertion of the theorem is equivalent to

$$
0=1-\frac{E_{34}}{E_{41}}+\frac{E_{29}}{E_{41}}+\frac{E_{1}}{E_{41}}-\frac{E_{1} E_{6} E_{15} E_{20} E_{29} E_{34} E_{36} E_{50} E_{14} E_{21} E_{35} E_{49}}{E_{2} E_{3} E_{12} E_{17} E_{18} E_{23} E_{32} E_{33} E_{37} E_{38} E_{47} E_{52}},
$$

where we used the notation (6.1) with $N=105$. Using (6.3), we see that the right-hand side is a modular function for the group $\Gamma_{1}(105)$. We estimate the sum of the orders of the right-hand side at the cusps using the same programmes as in the previous proof with modifications of the parameters. The upper bound on the order of the righthand side at $i \infty$ is also 148 , so it suffices to check that the first 148 coefficients of the right-hand side are zero in order to prove the identity, which we checked using a computer.

Proof of Theorem [3. It is elementary to see that squares that are congruent to 121 modulo 840 are of the form $S^{2}$, where $S \equiv 11,31,59,101,109,151,179,199(\bmod 210)$. If we now do a computation analogous to the one in the proof of Theorem 1, we obtain

$$
\begin{aligned}
\sum_{n=0}^{\infty}(-1)^{\lfloor(n+4) / 8\rfloor} q^{a_{n}}=\left(q^{105}, q^{47}, q^{58} ; q^{105}\right)_{\infty}+q\left(q^{105}, q^{37}, q^{68} ; q^{105}\right)_{\infty} \\
+q^{4}\left(q^{105}, q^{23}, q^{82} ; q^{105}\right)_{\infty}+q^{12}\left(q^{105}, q^{2}, q^{103} ; q^{105}\right)_{\infty} .
\end{aligned}
$$

Next, we observe that, using the notation (6.1) with $N=105$, the assertion of the theorem is equivalent to

$$
0=1+\frac{E_{37}}{E_{47}}+\frac{E_{23}}{E_{47}}+\frac{E_{2}}{E_{47}}-\frac{E_{2} E_{5} E_{7} E_{12} E_{23} E_{28} E_{30} E_{33} E_{35} E_{37} E_{40} E_{42}}{E_{1} E_{4} E_{6} E_{11} E_{24} E_{29} E_{31} E_{34} E_{36} E_{39} E_{41} E_{46}} .
$$

Using (6.3), we see that the right-hand side is a modular function for the group $\Gamma_{1}(105)$. We estimate the sum of the orders of the right-hand side at the cusps using the same programmes as before with modifications of the parameters. The upper bound on the order of the right-hand side at $i \infty$ is also 148, so it suffices to check that the first 148 coefficients of the right-hand side are zero in order to prove the identity, which we checked using a computer.

Proof of Theorem 4. It is elementary to see that squares that are congruent to 289 modulo 840 are of the form $S^{2}$, where $S \equiv 17,53,67,73,137,143,157,193(\bmod 210)$. If we now do a computation analogous to the one in the proof of Theorem 1, we obtain

$$
\begin{aligned}
\sum_{n=0}^{\infty}(-1)^{t(n)} q^{a_{n}}=\left(q^{105}, q^{44}, q^{61} ; q^{105}\right)_{\infty}+q^{3}\left(q^{105}, q^{26}, q^{79} ; q^{105}\right)_{\infty} & \\
& -q^{5}\left(q^{105}, q^{19}, q^{86} ; q^{105}\right)_{\infty}+q^{6}\left(q^{105}, q^{16}, q^{89} ; q^{105}\right)_{\infty} .
\end{aligned}
$$

Next, we observe that, using the notation (6.1) with $N=105$, the assertion of the theorem is equivalent to

$$
0=1+\frac{E_{26}}{E_{44}}-\frac{E_{19}}{E_{44}}+\frac{E_{16}}{E_{44}}-\frac{E_{5} E_{9} E_{14} E_{16} E_{19} E_{21} E_{26} E_{30} E_{35} E_{40} E_{49} E_{51}}{E_{3} E_{8} E_{13} E_{17} E_{18} E_{22} E_{27} E_{32} E_{38} E_{43} E_{48} E_{52}} .
$$

Using (6.3), we see that the right-hand side is a modular function for the group $\Gamma_{1}(105)$. We estimate the sum of the orders of the right-hand side at the cusps using the same programmes as before with modifications of the parameters. The upper bound on the order of the right-hand side at $i \infty$ is also 148, so it suffices to check that the first 
148 coefficients of the right-hand side are zero in order to prove the identity, which we checked using a computer.

Proof of Theorem 5. It is elementary to see that squares that are congruent to 1 modulo 840 are of the form $S^{2}$, where $S \equiv 1,29,41,71,139,169,181,209(\bmod 210)$. If we now do a computation analogous to the one in the proof of Theorem 1, we obtain

$$
\begin{aligned}
\sum_{n=0}^{\infty}(-1)^{\lfloor(n+4) / 8\rfloor} q^{a_{n}}=\left(q^{105}, q^{52}, q^{53} ; q^{105}\right)_{\infty}+q\left(q^{105}, q^{38}, q^{67} ; q^{105}\right)_{\infty} \\
+q^{2}\left(q^{105}, q^{32}, q^{73} ; q^{105}\right)_{\infty}+q^{6}\left(q^{105}, q^{17}, q^{88} ; q^{105}\right)_{\infty} .
\end{aligned}
$$

Next, we observe that, using the notation (6.1) with $N=105$, the assertion of the theorem is equivalent to

$$
0=1+\frac{E_{38}}{E_{52}}+\frac{E_{32}}{E_{52}}+\frac{E_{17}}{E_{52}}-\frac{E_{3} E_{7} E_{10} E_{17} E_{18} E_{25} E_{28} E_{32} E_{35} E_{38} E_{42} E_{45}}{E_{1} E_{6} E_{9} E_{16} E_{19} E_{26} E_{29} E_{34} E_{36} E_{41} E_{44} E_{51}} .
$$

Using (6.3), we see that the right-hand side is a modular function for the group $\Gamma_{1}(105)$. We estimate the sum of the orders of the right-hand side at the cusps using the same programmes as before with modifications of the parameters. The upper bound on the order of the right-hand side at $i \infty$ is also 148, so it suffices to check that the first 148 coefficients of the right-hand side are zero in order to prove the identity, which we checked using a computer.

Proof of Theorem 6. It is elementary to see that squares that are congruent to 169 modulo 840 are of the form $S^{2}$, where $S \equiv 13,43,83,97,113,127,167,197(\bmod 210)$. If we now do a computation analogous to the one in the proof of Theorem 1, we obtain

$$
\begin{aligned}
\sum_{n=0}^{\infty}(-1)^{t(n)} q^{a_{n}}=\left(q^{105}, q^{46}, q^{59}\right. & \left.; q^{105}\right)_{\infty}+q^{2}\left(q^{105}, q^{31}, q^{74} ; q^{105}\right)_{\infty} \\
& +q^{8}\left(q^{105}, q^{11}, q^{94} ; q^{105}\right)_{\infty}-q^{11}\left(q^{105}, q^{4}, q^{101} ; q^{105}\right)_{\infty}
\end{aligned}
$$

Next, we observe that, using the notation (6.1) with $N=105$, the assertion of the theorem is equivalent to

$$
0=1+\frac{E_{31}}{E_{46}}+\frac{E_{11}}{E_{46}}-\frac{E_{4}}{E_{46}}-\frac{E_{4} E_{10} E_{11} E_{14} E_{21} E_{24} E_{25} E_{31} E_{35} E_{39} E_{45} E_{49}}{E_{2} E_{8} E_{12} E_{13} E_{22} E_{23} E_{27} E_{33} E_{37} E_{43} E_{47} E_{48}} .
$$

Using (6.3), we see that the right-hand side is a modular function for the group $\Gamma_{1}(105)$. We estimate the sum of the orders of the right-hand side at the cusps using the same programmes as before with modifications of the parameters. The upper bound on the order of the right-hand side at $i \infty$ is also 148, so it suffices to check that the first 148 coefficients of the right-hand side are zero in order to prove the identity, which we checked using a computer.

Proof of Theorem 7 . It is elementary to see that squares that are congruent to 1 modulo 240 are of the form $S^{2}$, where $S \equiv 1,31,41,49,71,79,89,119(\bmod 120)$. If we now do 
a computation analogous to the one in the proof of Theorem 1, we obtain

$$
\begin{aligned}
\sum_{n=0}^{\infty}(-1)^{\lfloor(n+2) / 4\rfloor} q^{a_{n}} & =\left(q^{120},-q^{59},-q^{61} ; q^{120}\right)_{\infty}+q^{4}\left(q^{120},-q^{29},-q^{91} ; q^{120}\right)_{\infty} \\
& -q^{7}\left(q^{120},-q^{19},-q^{101} ; q^{120}\right)_{\infty}-q^{10}\left(q^{120},-q^{11},-q^{109} ; q^{120}\right)_{\infty}
\end{aligned}
$$

Rewriting this expression, we see that the assertion of the theorem is equivalent to

$$
\begin{gathered}
0=\frac{\left(q^{120} ; q^{120}\right)_{\infty}\left(q^{118}, q^{122} ; q^{240}\right)_{\infty}}{\left(q^{59}, q^{61} ; q^{120}\right)_{\infty}}+q^{4} \frac{\left(q^{120} ; q^{120}\right)_{\infty}\left(q^{58}, q^{182} ; q^{240}\right)_{\infty}}{\left(q^{29}, q^{91} ; q^{120}\right)_{\infty}} \\
-q^{7} \frac{\left(q^{120} ; q^{120}\right)_{\infty}\left(q^{22}, q^{218} ; q^{240}\right)_{\infty}}{\left(q^{11}, q^{109} ; q^{120}\right)_{\infty}}-q^{10} \frac{\left(q^{120} ; q^{120}\right)_{\infty}\left(q^{22}, q^{218} ; q^{240}\right)_{\infty}}{\left(q^{11}, q^{109} ; q^{120}\right)_{\infty}} \\
-\frac{\left(q, q^{7}, q^{8} ; q^{8}\right)_{\infty}\left(q^{6}, q^{10} ; q^{16}\right)_{\infty}}{\left(q, q^{4} ; q^{5}\right)_{\infty}}
\end{gathered}
$$

By dividing both sides of the identity by the first term on the right-hand side and using the notation (6.1) with $N=240$, we obtain

$$
\begin{aligned}
0=1+\frac{E_{58} E_{59} E_{61}}{E_{29} E_{91} E_{118}} & -\frac{E_{38} E_{59} E_{61}}{E_{19} E_{101} E_{118}}-\frac{E_{22} E_{59} E_{61}}{E_{11} E_{109} E_{118}} \\
-\frac{E_{7} E_{8} E_{10} E_{15} E_{17} E_{22} E_{23} E_{25} E_{32} E_{33} E_{38} E_{40} E_{42} E_{47} E_{48} E_{55} E_{57} E_{58}}{E_{4} E_{11} E_{14} E_{19} E_{21} E_{29} E_{34} E_{36} E_{44} E_{46} E_{51}} & \times \frac{E_{63} E_{65} E_{70} E_{72} E_{73} E_{80} E_{87} E_{88} E_{90} E_{95} E_{97} E_{102} E_{103} E_{105} E_{112} E_{113}}{E_{66} E_{69} E_{76} E_{84} E_{91} E_{94} E_{99} E_{101} E_{109} E_{114} E_{116}} .
\end{aligned}
$$

Each term on the right-hand side is a modular function for the group $\Gamma_{1}(240)$. As before, using Magma we compute a list of all the cusps. Here we have 448 cusps. Again, we can give an upper bound on the order of the right-hand side at $i \infty$. Running our programme, we obtain 592. We need to verify that the right-hand side has the form $0+0 q+\cdots+0 q^{592}+\cdots$, which can be routinely done. This proves the identity.

Proof of Theorem 8. It is elementary to see that squares that are congruent to 49 modulo 240 are of the form $S^{2}$, where $S \equiv 7,17,23,47,73,97,103,113(\bmod 120)$. If we now do a computation analogous to the one in the proof of Theorem 1, we obtain

$$
\begin{aligned}
\sum_{n=0}^{\infty}(-1)^{\lfloor 5 n / 4\rfloor} q^{a_{n}}=\left(q^{120},-q^{53},-q^{67} ; q^{120}\right)_{\infty}-q\left(q^{120},-q^{43},-q^{77} ; q^{120}\right)_{\infty} \\
+q^{2}\left(q^{120},-q^{37},-q^{83} ; q^{120}\right)_{\infty}-q^{9}\left(q^{120},-q^{13},-q^{107} ; q^{120}\right)_{\infty} \\
=\frac{\left(q^{120} ; q^{120}\right)_{\infty}\left(q^{106}, q^{134} ; q^{240}\right)_{\infty}}{\left(q^{53}, q^{67} ; q^{120}\right)_{\infty}}-q \frac{\left(q^{120} ; q^{120}\right)_{\infty}\left(q^{86}, q^{154} ; q^{240}\right)_{\infty}}{\left(q^{43}, q^{77} ; q^{120}\right)_{\infty}} \\
+q^{2} \frac{\left(q^{120} ; q^{120}\right)_{\infty}\left(q^{74}, q^{166} ; q^{240}\right)_{\infty}}{\left(q^{37}, q^{83} ; q^{120}\right)_{\infty}}-q^{9} \frac{\left(q^{120} ; q^{120}\right)_{\infty}\left(q^{26}, q^{214} ; q^{240}\right)_{\infty}}{\left(q^{13}, q^{107} ; q^{120}\right)_{\infty}}
\end{aligned}
$$


Next, we observe that, using the notation (6.1) with $N=240$, the assertion of the theorem is equivalent to

$$
\begin{gathered}
0=1-\frac{E_{53} E_{67} E_{86}}{E_{43} E_{77} E_{106}}+\frac{E_{53} E_{67} E_{74}}{E_{37} E_{83} E_{106}}-\frac{E_{26} E_{53} E_{67}}{E_{13} E_{106} E_{107}} \\
-\frac{E_{1} E_{6} E_{9} E_{10} E_{15} E_{16} E_{24} E_{25} E_{26} E_{31} E_{39} E_{40} E_{41} E_{49} E_{54} E_{55} E_{56}}{E_{2} E_{3} E_{12} E_{13} E_{18} E_{27} E_{28} E_{37} E_{43} E_{52}} \\
\quad \times \frac{E_{64} E_{65} E_{70} E_{71} E_{74} E_{79} E_{80} E_{81} E_{86} E_{89} E_{90} E_{95} E_{96} E_{104} E_{105} E_{111} E_{119}}{E_{62} E_{68} E_{77} E_{78} E_{82} E_{83} E_{92} E_{93} E_{98} E_{107} E_{108} E_{117}} .
\end{gathered}
$$

As before, each term is a modular function for the group $\Gamma_{1}(240)$. The upper bound on the order of the right-hand side at $i \infty$ is 592. We verified that the right-hand side has the form $0+0 q+\cdots+0 q^{592}+\cdots$. This proves the theorem.

Proof of Theorem 9. It is elementary to see that squares that are congruent to 121 modulo 240 are of the form $S^{2}$, where $S \equiv 11,19,29,59,61,91,101,109(\bmod 120)$. If we now do a computation analogous to the one in the proof of Theorem 1, we obtain

$$
\begin{gathered}
\sum_{n=0}^{\infty}(-1)^{\lfloor(n+2) / 4\rfloor} q^{a_{n}}=\left(q^{120},-q^{49},-q^{71} ; q^{120}\right)_{\infty}+q\left(q^{120},-q^{41},-q^{79} ; q^{120}\right)_{\infty} \\
-q^{3}\left(q^{120},-q^{31},-q^{89} ; q^{120}\right)_{\infty}-q^{14}\left(q^{120},-q,-q^{119} ; q^{120}\right)_{\infty} \\
=\frac{\left(q^{120} ; q^{120}\right)_{\infty}\left(q^{98}, q^{142} ; q^{240}\right)_{\infty}}{\left(q^{49}, q^{71} ; q^{120}\right)_{\infty}}+q \frac{\left(q^{120} ; q^{120}\right)_{\infty}\left(q^{82}, q^{158} ; q^{240}\right)_{\infty}}{\left(q^{41}, q^{79} ; q^{120}\right)_{\infty}} \\
-q^{3} \frac{\left(q^{120} ; q^{120}\right)_{\infty}\left(q^{62}, q^{178} ; q^{240}\right)_{\infty}}{\left(q^{31}, q^{89} ; q^{120}\right)_{\infty}}-q^{14} \frac{\left(q^{120} ; q^{120}\right)_{\infty}\left(q^{2}, q^{238} ; q^{240}\right)_{\infty}}{\left(q^{1}, q^{119} ; q^{120}\right)_{\infty}}
\end{gathered}
$$

Next, we observe that, using the notation (6.1) with $N=240$, the assertion of the theorem is equivalent to

$$
\begin{gathered}
0=1+\frac{E_{49} E_{71} E_{82}}{E_{41} E_{79} E_{98}}-\frac{E_{49} E_{62} E_{71}}{E_{31} E_{89} E_{98}}-\frac{E_{2} E_{49} E_{71}}{E_{1} E_{98} E_{119}} \\
-\frac{E_{2} E_{3} E_{5} E_{8} E_{13} E_{18} E_{27} E_{30} E_{32} E_{35} E_{37} E_{40} E_{43} E_{45} E_{48} E_{50} E_{53}}{E_{1} E_{4} E_{6} E_{9} E_{26} E_{31} E_{36} E_{39} E_{41} E_{44} E_{54}} \\
\times \frac{E_{62} E_{67} E_{72} E_{75} E_{77} E_{78} E_{80} E_{82} E_{83} E_{85} E_{88} E_{93} E_{107} E_{110} E_{112} E_{115} E_{117}}{E_{74} E_{76} E_{79} E_{81} E_{84} E_{86} E_{89} E_{106} E_{111} E_{116} E_{119}} .
\end{gathered}
$$

As before, each term is a modular function for the group $\Gamma_{1}(240)$. The upper bound on the order of the right-hand side at $i \infty$ is 592 . We verified that the right-hand side has the form $0+0 q+\cdots+0 q^{592}+\cdots$. This proves the theorem.

Proof of Theorem 10. It is elementary to see that squares that are congruent to 169 modulo 240 are of the form $S^{2}$, where $S \equiv 13,37,43,53,67,77,83,107(\bmod 120)$. If 
we now do a computation analogous to the one in the proof of Theorem 1, we obtain

$$
\begin{aligned}
\sum_{n=0}^{\infty}(-1)^{\lfloor 5 n / 4\rfloor} q^{a_{n}}=\left(q^{120},-q^{47},-q^{73} ; q^{120}\right)_{\infty}-q^{5}\left(q^{120},-q^{23},-q^{97} ; q^{120}\right)_{\infty} \\
\quad+q^{7}\left(q^{120},-q^{17},-q^{103} ; q^{120}\right)_{\infty}-q^{11}\left(q^{120},-q^{7},-q^{113} ; q^{120}\right)_{\infty} \\
=\frac{\left(q^{120} ; q^{120}\right)_{\infty}\left(q^{94}, q^{146} ; q^{240}\right)_{\infty}}{\left(q^{47}, q^{73} ; q^{120}\right)_{\infty}}-q^{5} \frac{\left(q^{120} ; q^{120}\right)_{\infty}\left(q^{46}, q^{194} ; q^{240}\right)_{\infty}}{\left(q^{23}, q^{97} ; q^{120}\right)_{\infty}} \\
+q^{7} \frac{\left(q^{120} ; q^{120}\right)_{\infty}\left(q^{34}, q^{206} ; q^{240}\right)_{\infty}}{\left(q^{17}, q^{103} ; q^{120}\right)_{\infty}}-q^{11} \frac{\left(q^{120} ; q^{120}\right)_{\infty}\left(q^{14}, q^{226} ; q^{240}\right)_{\infty}}{\left(q^{7}, q^{113} ; q^{120}\right)_{\infty}}
\end{aligned}
$$

Next, we observe that, using the notation (6.1) with $N=240$, the assertion of the theorem is equivalent to

$$
\begin{gathered}
0=1-\frac{E_{46} E_{47} E_{73}}{E_{23} E_{94} E_{97}}+\frac{E_{34} E_{47} E_{73}}{E_{17} E_{94} E_{103}}-\frac{E_{14} E_{47} E_{73}}{E_{7} E_{94} E_{113}} \\
-\frac{E_{5} E_{11} E_{14} E_{16} E_{19} E_{21} E_{24} E_{29} E_{30} E_{34} E_{35} E_{40} E_{45} E_{46} E_{50} E_{51} E_{56} E_{59}}{E_{7} E_{12} E_{17} E_{22} E_{23} E_{28} E_{33} E_{38} E_{42} E_{52} E_{57} E_{58}} \\
\times \frac{E_{61} E_{64} E_{66} E_{69} E_{75} E_{80} E_{85} E_{91} E_{96} E_{99} E_{101} E_{104} E_{109} E_{110} E_{114} E_{115}}{E_{63} E_{68} E_{87} E_{92} E_{97} E_{102} E_{103} E_{108} E_{113} E_{118}} .
\end{gathered}
$$

As before, each term is a modular function for the group $\Gamma_{1}(240)$. The upper bound on the order of the right-hand side at $i \infty$ is 592. We verified that the right-hand side has the form $0+0 q+\cdots+0 q^{592}+\cdots$. This proves the theorem.

Proof of Theorem 11. This is a special case of Theorem 36.

Proof of Theorem 12. This is a special case of Theorem 36.

Proof of Theorem 13. This is a direct consequence of the Jacobi triple product identity (4.1): one replaces $q$ by $q^{10}$ and then chooses $z=-q^{6}$ there.

Proof of Theorem 14. Using (4.3), we obtain

$$
\sum_{n=0}^{\infty}\left(q^{n(n+1)}-q^{5 n(n+1)+1}\right)=\left(q^{2} ; q^{2}\right)_{\infty}\left(-q^{2} ; q^{2}\right)_{\infty}^{2}-q\left(q^{10} ; q^{10}\right)_{\infty}\left(-q^{10} ; q^{10}\right)_{\infty}^{2}
$$

Hence, the assertion of the theorem is equivalent to

$$
0=\frac{\left(q^{4} ; q^{4}\right)_{\infty}^{2}}{\left(q^{2} ; q^{2}\right)_{\infty}}-q \frac{\left(q^{20} ; q^{20}\right)_{\infty}^{2}}{\left(q^{10} ; q^{10}\right)_{\infty}}-\frac{\left(q, q^{9}, q^{10} ; q^{10}\right)_{\infty}\left(q^{8}, q^{12} ; q^{20}\right)_{\infty}}{\left(q^{2}, q^{3} ; q^{5}\right)_{\infty}}
$$

Using the notation (6.1) with $N=20$, we see that this is equivalent to

$$
0=1-\frac{E_{2} E_{6}}{E_{4} E_{8}}-\frac{E_{1} E_{6} E_{9} E_{10}}{E_{3} E_{4} E_{7} E_{8}}
$$

The right-hand side is a modular function for the group $\Gamma_{1}(20)$. The cusps for this group are computed as usual using Magma:

$$
\left\{\infty, 0, \frac{1}{7}, \frac{3}{20}, \frac{1}{6}, \frac{2}{11}, \frac{1}{5}, \frac{1}{4}, \frac{3}{10}, \frac{1}{3}, \frac{7}{20}, \frac{11}{30}, \frac{3}{8}, \frac{2}{5}, \frac{9}{20}, \frac{1}{2}, \frac{7}{12}, \frac{3}{5}, \frac{3}{4}, \frac{4}{5}\right\} .
$$


There are in total 20 cusps. Estimating the order of the right-hand side at $i \infty$, one obtains an upper bound of 4 . Hence it is sufficient to show that the right-hand side has the form $0+0 q+0 q^{2}+0 q^{3}+0 q^{4}+\cdots$, which can be done routinely.

Proof of Theorem 15. Using (4.2), we obtain

$$
1+\sum_{n=1}^{\infty}\left(q^{n^{2}}+q^{5 n^{2}}\right)=\frac{1}{2}\left(\left(q^{2} ; q^{2}\right)_{\infty}\left(-q ; q^{2}\right)_{\infty}^{2}+\left(q^{10} ; q^{10}\right)_{\infty}\left(-q^{5} ; q^{10}\right)_{\infty}^{2}\right) .
$$

Hence, the assertion of the theorem is equivalent to

$$
0=\frac{1}{2} \frac{\left(q^{2} ; q^{2}\right)_{\infty}\left(q^{2} ; q^{4}\right)_{\infty}^{2}}{\left(q ; q^{2}\right)_{\infty}^{2}}+\frac{1}{2} \frac{\left(q^{10} ; q^{10}\right)_{\infty}\left(q^{10} ; q^{20}\right)_{\infty}^{2}}{\left(q^{5} ; q^{10}\right)_{\infty}^{2}}-\frac{\left(q^{2}, q^{8}, q^{10} ; q^{10}\right)_{\infty}\left(q^{6}, q^{14} ; q^{20}\right)_{\infty}}{\left(q, q^{4} ; q^{5}\right)_{\infty}}
$$

Using the notation (6.1) with $N=20$, we see that this is equivalent to

$$
0=\frac{1}{2}+\frac{1}{2} \frac{E_{1}^{2} E_{3}^{2} E_{7}^{2} E_{9}^{2}}{E_{2}^{3} E_{4} E_{6}^{3} E_{8}}-\frac{E_{1} E_{3}^{2} E_{5}^{2} E_{7}^{2} E_{9}}{E_{2}^{2} E_{4}^{2} E_{6}^{3} E_{10}} .
$$

The right-hand side is a modular function for the group $\Gamma_{1}(20)$. There are in total the 20 cusps exhibited in the previous proof. Estimating the order of the right-hand side at $i \infty$, one obtains an upper bound of 4 . Hence it is sufficient to show that the righthand side has the form $0+0 q+0 q^{2}+0 q^{3}+0 q^{4}+\cdots$, which can be done routinely.

Proof of Theorem 16. This is a direct consequence of the Jacobi triple product identity (4.1): one replaces $q$ by $q^{10}$ and then chooses $z=-q^{7}$ there.

Proof of Theorem 17. Using (4.3), we obtain

$$
\sum_{n=0}^{\infty}\left(q^{n(n+1)}+q^{5 n(n+1)+1}\right)=\left(q^{2} ; q^{2}\right)_{\infty}\left(-q^{2} ; q^{2}\right)_{\infty}^{2}+q\left(q^{10} ; q^{10}\right)_{\infty}\left(-q^{10} ; q^{10}\right)_{\infty}^{2}
$$

Hence, the assertion of the theorem is equivalent to

$$
0=\frac{\left(q^{4} ; q^{4}\right)_{\infty}^{2}}{\left(q^{2} ; q^{2}\right)_{\infty}}+q \frac{\left(q^{20} ; q^{20}\right)_{\infty}^{2}}{\left(q^{10} ; q^{10}\right)_{\infty}}-\frac{\left(q^{3}, q^{7}, q^{10} ; q^{10}\right)_{\infty}\left(q^{4}, q^{16} ; q^{20}\right)_{\infty}}{\left(q, q^{4} ; q^{5}\right)_{\infty}}
$$

Using the notation (6.1) with $N=20$, we see that this is equivalent to

$$
0=1+\frac{E_{2} E_{6}}{E_{4} E_{8}}-\frac{E_{2} E_{3} E_{7} E_{10}}{E_{1} E_{4} E_{8} E_{9}} .
$$

The right-hand side is a modular function for the group $\Gamma_{1}(20)$. There are in total the 20 cusps exhibited in the proof of Theorem 15. Estimating the order of the righthand side at $i \infty$, one obtains an upper bound of 4 . Hence it is sufficient to show that the right-hand side has the form $0+0 q+0 q^{2}+0 q^{3}+0 q^{4}+\cdots$, which can be done routinely.

Proof of Theorem 18. This is a direct consequence of the Jacobi triple product identity (4.1): one replaces $q$ by $q^{10}$ and then chooses $z=-q^{8}$ there.

Proof of Theorem 19. This is a direct consequence of the Jacobi triple product identity (4.1): one replaces $q$ by $q^{10}$ and then chooses $z=-q^{9}$ there. 
Proof of Theorem 20, Using (4.2), we obtain

$$
\sum_{n=1}^{\infty}\left(q^{n^{2}-1}-q^{5 n^{2}-1}\right)=\frac{1}{2 q}\left(\left(q^{2} ; q^{2}\right)_{\infty}\left(-q ; q^{2}\right)_{\infty}^{2}-\left(q^{10} ; q^{10}\right)_{\infty}\left(-q^{5} ; q^{10}\right)_{\infty}^{2}\right) .
$$

Hence, the assertion of the theorem is equivalent to

$$
0=\frac{1}{2 q} \frac{\left(q^{2} ; q^{2}\right)_{\infty}\left(q^{2} ; q^{4}\right)_{\infty}^{2}}{\left(q ; q^{2}\right)_{\infty}^{2}}-\frac{1}{2 q} \frac{\left(q^{10} ; q^{10}\right)_{\infty}\left(q^{10} ; q^{20}\right)_{\infty}^{2}}{\left(q^{5} ; q^{10}\right)_{\infty}^{2}}-\frac{\left(q^{4}, q^{6}, q^{10} ; q^{10}\right)_{\infty}\left(q^{2}, q^{18} ; q^{20}\right)_{\infty}}{\left(q^{2} ; q^{3} ; q^{5}\right)_{\infty}}
$$

Using the notation (6.1) with $N=20$, we see that this is equivalent to

$$
0=\frac{1}{2}-\frac{1}{2} \frac{E_{1}^{2} E_{3}^{2} E_{7}^{2} E_{9}^{2}}{E_{2}^{3} E_{4} E_{6}^{3} E_{8}}-\frac{E_{1}^{2} E_{3} E_{5}^{2} E_{7} E_{9}^{2}}{E_{2}^{3} E_{6}^{2} E_{8}^{2} E_{10}} .
$$

The right-hand side is a modular function for the group $\Gamma_{1}(20)$. There are in total the 20 cusps exhibited in the proof of Theorem 15. Estimating the order of the righthand side at $i \infty$, one obtains an upper bound of 4 . Hence it is sufficient to show that the right-hand side has the form $0+0 q+0 q^{2}+0 q^{3}+0 q^{4}+\cdots$, which can be done routinely.

Proof of Theorem 21. This is a special case of Corollary 35,

Proof of Theorem 22. This is a special case of Theorem 34.

Proof of Theorem [23. This is a special case of Corollary 35.

Proof of Theorem 24. This is a special case of Theorem 34 .

Proof of Theorem 25. This is a special case of Theorem 34.

Proof of Theorem [26. It is elementary to see that squares that are congruent to 1 modulo 48 are of the form $N^{2}$, where $N \equiv 1,7,17,23(\bmod 24)$. If we now do a computation analogous to the one in the proof of Theorem 1, we obtain

$$
\sum_{n=0}^{\infty}(-1)^{\lfloor(n+2) / 4\rfloor} q^{a_{n}}=\left(q^{24}, q^{13}, q^{11} ; q^{24}\right)_{\infty}+q\left(q^{24}, q^{19}, q^{5} ; q^{24}\right)_{\infty}
$$

Next, we observe that, using the notation (6.1) with $N=24$, the assertion of the theorem is equivalent to

$$
0=1+\frac{E_{5}}{E_{11}}-\frac{E_{2} E_{6} E_{8} E_{10}}{E_{1} E_{7} E_{9} E_{11}} .
$$

The right-hand side is a modular function for the group $\Gamma_{1}(24)$. The cusps for this group are computed as usual using Magma:

$$
\left\{\infty, 0, \frac{1}{8}, \frac{1}{7}, \frac{1}{6}, \frac{2}{11}, \frac{1}{5}, \frac{5}{24}, \frac{3}{14}, \frac{2}{9}, \frac{1}{4}, \frac{7}{24}, \frac{11}{36}, \frac{1}{3}, \frac{3}{8}, \frac{7}{18}, \frac{5}{12}, \frac{4}{9}, \frac{11}{24}, \frac{1}{2}, \frac{9}{16}, \frac{5}{8}, \frac{2}{3}, \frac{3}{4}\right\} .
$$

There are in total 24 cusps. Estimating the order of the right-hand side at $i \infty$, one obtains an upper bound of 4 . Hence it is sufficient to show that the right-hand side has the form $0+0 q+0 q^{2}+0 q^{3}+0 q^{4}+\cdots$, which can be done routinely. 
Proof of Theorem 27. It is elementary to see that squares that are congruent to 25 modulo 48 are of the form $S^{2}$, where $S \equiv 5,11,13,19(\bmod 24)$. If we now do a computation analogous to the one in the proof of Theorem 1, we obtain

$$
\sum_{n=0}^{\infty}(-1)^{\lfloor 5 n / 4\rfloor} q^{a_{n}}=\left(q^{24}, q^{17}, q^{7} ; q^{24}\right)_{\infty}-q^{2}\left(q^{24}, q^{23}, q ; q^{24}\right)_{\infty} .
$$

Next, we observe that, using the notation (6.1) with $N=24$, the assertion of the theorem is equivalent to

$$
0=1-\frac{E_{1}}{E_{7}}-\frac{E_{2} E_{6} E_{8} E_{10}}{E_{3} E_{5} E_{7} E_{11}} .
$$

The right-hand side is a modular function for the group $\Gamma_{1}(24)$. There are in total the 24 cusps exhibited in the previous proof. Estimating the order of the right-hand side at $i \infty$, one obtains an upper bound of 4 . Hence it is sufficient to show that the righthand side has the form $0+0 q+0 q^{2}+0 q^{3}+0 q^{4}+\cdots$, which can be done routinely.

Proof of Theorem 28. This is a special case of Theorem 36.

Proof of Theorem 29. This is a special case of Theorem 36.

Proof of Theorem 30. This theorem is a special case of Corollary 37.

Proof of Theorem 31. This is a special case of Theorem 38.

Proof of Theorem 32. This is a special case of Theorem 38.

\section{Theta FUnCtion IDENTITIES}

The purpose of this section is, first of all, to present Weierstraß' addition formula for theta functions, and, second, to make two special cases explicit that are particularly used in the proofs of our theorems from Sections 2 and 3 given in the next section, and also in the proofs in Section 10.

In this section and the following ones, we use a different notation for the theta functions that appear in our context, namely

$$
\theta(\alpha ; q):=(\alpha, q / \alpha ; q)_{\infty}
$$

It should be noted that, up to a power of $q$, the function $E_{g}(q ; N)$ that we used in Sections 6 and 7 can be expressed as $\theta\left(q^{g} ; q^{N}\right)$.

Using the above notation, Weierstraß' addition formula (cf. [7, p. 451, Example 5]) reads

$$
\begin{aligned}
\theta(x y ; q) \theta(x / y ; q) \theta(u v ; q) \theta(u / v ; q)-\theta(x v ; q) \theta(x / v ; q) \theta(u y ; q) \theta(u / y ; q) \\
=\frac{u}{y} \theta(y v ; q) \theta(y / v ; q) \theta(x u ; q) \theta(x / u ; q) .
\end{aligned}
$$

Two specialisations of this formula are of particular importance in our context. If, in (8.1), we replace $q$ by $q^{3 N}$ and specialise $x=q^{N}, y=u^{2} / q^{N}$, and $v=q^{N} / u$, then we 
obtain the relation

$$
\begin{aligned}
& \theta\left(u^{2} ; q^{3 N}\right) \theta\left(q^{2 N} / u^{2} ; q^{3 N}\right) \theta\left(q^{N} ; q^{3 N}\right) \theta\left(u^{2} / q^{N} ; q^{3 N}\right) \\
& -\theta\left(q^{2 N} / u ; q^{3 N}\right) \theta\left(u ; q^{3 N}\right) \theta\left(u^{3} / q^{N} ; q^{3 N}\right) \theta\left(q^{N} / u ; q^{3 N}\right) \\
& \quad=\frac{q^{N}}{u} \theta\left(u ; q^{3 N}\right) \theta\left(u^{3} / q^{2 N} ; q^{3 N}\right) \theta\left(q^{N} u ; q^{3 N}\right) \theta\left(q^{N} / u ; q^{3 N}\right),
\end{aligned}
$$

or, equivalently,

$$
\begin{aligned}
\theta\left(u^{3} / q^{N} ; q^{3 N}\right)+\frac{q^{N}}{u} \theta\left(u^{3} / q^{2 N} ; q^{3 N}\right) & \\
& =\frac{\theta\left(u^{2} ; q^{3 N}\right) \theta\left(q^{2 N} / u^{2} ; q^{3 N}\right) \theta\left(q^{N} ; q^{3 N}\right) \theta\left(u^{2} / q^{N} ; q^{3 N}\right)}{\theta\left(u ; q^{3 N}\right) \theta\left(q^{N} / u ; q^{3 N}\right) \theta\left(q^{N} u ; q^{3 N}\right)} .
\end{aligned}
$$

Written in alternative notation, this is

$$
\begin{aligned}
\left(u^{3} / q^{N}, q^{4 N} / u^{3}, q^{3 N} ; q^{3 N}\right)_{\infty}+\frac{q^{N}}{u}\left(u^{3} / q^{2 N}, q^{5 N} / u^{3}, q^{3 N} ; q^{3 N}\right)_{\infty} & \\
& =\frac{\left(u^{2} / q^{N}, q^{2 N} / u^{2}, q^{N} ; q^{N}\right)_{\infty}}{\left(u, q^{N} / u ; q^{N}\right)_{\infty}} .
\end{aligned}
$$

Similarly, if in (8.1) we replace $q$ by $q^{3 N}$ and specialise $x=q^{2 N}, y=u^{2} / q^{2 N}$, and $v=q^{2 N} / u$, then we obtain the relation

$$
\begin{aligned}
\theta\left(u^{2} ; q^{3 N}\right) \theta\left(q^{4 N} / u^{2} ; q^{3 N}\right) & \theta\left(q^{2 N} ; q^{3 N}\right) \theta\left(u^{2} / q^{2 N} ; q^{3 N}\right) \\
-\theta\left(q^{4 N} / u\right. & \left.; q^{3 N}\right) \theta\left(u ; q^{3 N}\right) \theta\left(u^{3} / q^{2 N} ; q^{3 N}\right) \theta\left(q^{2 N} / u ; q^{3 N}\right) \\
& =\frac{q^{2 N}}{u} \theta\left(u ; q^{3 N}\right) \theta\left(u^{3} / q^{4 N} ; q^{3 N}\right) \theta\left(q^{2 N} u ; q^{3 N}\right) \theta\left(q^{2 N} / u ; q^{3 N}\right),
\end{aligned}
$$

or, equivalently,

$$
\begin{aligned}
\theta\left(u^{3} / q^{2 N} ; q^{3 N}\right)-\frac{q^{3 N}}{u^{2}} \theta\left(u^{3} / q^{4 N}\right. & \left.; q^{3 N}\right) \\
& =\frac{\theta\left(u^{2} ; q^{3 N}\right) \theta\left(q^{4 N} / u^{2} ; q^{3 N}\right) \theta\left(q^{2 N} ; q^{3 N}\right) \theta\left(u^{2} / q^{2 N} ; q^{3 N}\right)}{\theta\left(u ; q^{3 N}\right) \theta\left(q^{2 N} / u ; q^{3 N}\right) \theta\left(u / q^{N} ; q^{3 N}\right)} .
\end{aligned}
$$

Written in alternative notation, this is

$$
\begin{aligned}
\left(u^{3} / q^{2 N}, q^{5 N} / u^{3}, q^{3 N} ; q^{3 N}\right)_{\infty}-\frac{q^{3 N}}{u^{2}}\left(u^{3} / q^{4 N}, q^{7 N} / u^{3}, q^{3 N} ; q^{3 N}\right)_{\infty} & \\
& =\frac{\left(u^{2} / q^{2 N}, q^{3 N} / u^{2}, q^{N} ; q^{N}\right)_{\infty}}{\left(u / q^{N}, q^{2 N} / u ; q^{N}\right)_{\infty}} .
\end{aligned}
$$

\section{Proofs by using the Weierstrass Relation}

In this section, we provide proofs of the theorems in Sections 2 and 3 that utilise the Weierstraß relation (8.1). Again, for the theorems which are specialisations of the parametric theorems in Section 10, we refer to the proofs given there (which also make use the Weierstraß relation). 
Proof of Theorem 1. Our point of departure is (7.2). By (8.2) with $N=35$ and $u=q^{26}$, respectively with $N=35$ and $u=q^{19}$, we get

$$
\sum_{n=0}^{\infty}(-1)^{t(n)} q^{a_{n}}=\frac{\left(q^{17}, q^{18}, q^{35} ; q^{35}\right)_{\infty}}{\left(q^{26}, q^{9} ; q^{35}\right)_{\infty}}+q^{4} \frac{\left(q^{3}, q^{32}, q^{35} ; q^{35}\right)_{\infty}}{\left(q^{19}, q^{16} ; q^{35}\right)_{\infty}} .
$$

If we now replace $q$ by $q^{35}$ and choose $u=q^{10}, v=q^{3}, x=q^{14}$, and $y=q^{6}$ in (8.1), we obtain ${ }^{2}$

$$
\begin{array}{r}
\theta\left(q^{17} ; q^{35}\right) \theta\left(q^{11} ; q^{35}\right) \theta\left(q^{16} ; q^{35}\right) \theta\left(q^{4} ; q^{35}\right)+ \\
+q^{4} \theta\left(q^{9} ; q^{35}\right) \theta\left(q^{3} ; q^{35}\right) \theta\left(q^{24} ; q^{35}\right) \theta\left(q^{4} ; q^{35}\right) \\
=\theta\left(q^{20} ; q^{35}\right) \theta\left(q^{8} ; q^{35}\right) \theta\left(q^{13} ; q^{35}\right) \theta\left(q^{7} ; q^{35}\right)
\end{array}
$$

and thus the above right-hand side becomes

$$
\frac{\theta\left(q^{20} ; q^{35}\right) \theta\left(q^{8} ; q^{35}\right) \theta\left(q^{13} ; q^{35}\right) \theta\left(q^{7} ; q^{35}\right)\left(q^{35} ; q^{35}\right)_{\infty}}{\theta\left(q^{16} ; q^{35}\right) \theta\left(q^{9} ; q^{35}\right) \theta\left(q^{11} ; q^{35}\right) \theta\left(q^{4} ; q^{35}\right)}
$$

which is equivalent to the right-hand side of (2.1).

Proof of Theorem 2. Our point of departure is (7.4). By (8.2) with $N=35$ and $u=q^{33}$, respectively with $N=35$ and $u=q^{23}$, we get

$$
\sum_{n=0}^{\infty}(-1)^{t(n)} q^{a_{n}}=\frac{\left(q^{31}, q^{4}, q^{35} ; q^{35}\right)_{\infty}}{\left(q^{33}, q^{2} ; q^{35}\right)_{\infty}}-q \frac{\left(q^{11}, q^{24}, q^{35} ; q^{35}\right)_{\infty}}{\left(q^{23}, q^{12} ; q^{35}\right)_{\infty}} .
$$

If we now replace $q$ by $q^{35}$ and choose $u=q^{15}, v=q^{3}, x=q^{17}$, and $y=q^{14}$ in (8.1), we obtain

$$
\begin{aligned}
\theta\left(q^{31} ; q^{35}\right) \theta\left(q^{3} ; q^{35}\right) \theta\left(q^{18} ; q^{35}\right) \theta\left(q^{12} ; q^{35}\right) & -\theta\left(q^{20} ; q^{35}\right) \theta\left(q^{14} ; q^{35}\right) \theta\left(q^{29} ; q^{35}\right) \theta\left(q ; q^{35}\right) \\
& =q \theta\left(q^{17} ; q^{35}\right) \theta\left(q^{11} ; q^{35}\right) \theta\left(q^{32} ; q^{35}\right) \theta\left(q^{2} ; q^{35}\right)
\end{aligned}
$$

and thus the above right-hand side becomes

$$
\frac{\theta\left(q^{20} ; q^{35}\right) \theta\left(q^{14} ; q^{35}\right) \theta\left(q^{29} ; q^{35}\right) \theta\left(q ; q^{35}\right)\left(q^{35} ; q^{35}\right)_{\infty}}{\theta\left(q^{17} ; q^{35}\right) \theta\left(q^{12} ; q^{35}\right) \theta\left(q^{32} ; q^{35}\right) \theta\left(q^{2} ; q^{35}\right)},
$$

which is equivalent to the right-hand side of (2.2).

Proof of Theorem 3. Our point of departure is (7.5). By (8.2) with $N=35$ and $u=q^{31}$, respectively with $N=35$ and $u=q^{24}$, we get

$$
\sum_{n=0}^{\infty}(-1)^{\lfloor(n+4) / 8\rfloor} q^{a_{n}}=\frac{\left(q^{27}, q^{8}, q^{35} ; q^{35}\right)_{\infty}}{\left(q^{31}, q^{4} ; q^{35}\right)_{\infty}}+q \frac{\left(q^{13}, q^{22}, q^{35} ; q^{35}\right)_{\infty}}{\left(q^{24}, q^{11} ; q^{35}\right)_{\infty}} .
$$

If we now replace $q$ by $q^{35}$ and choose $u=q^{14}, v=q^{9}, x=q^{15}$, and $y=q^{13}$ in (8.1), we obtain

$$
\begin{array}{r}
\theta\left(q^{28} ; q^{35}\right) \theta\left(q^{2} ; q^{35}\right) \theta\left(q^{23} ; q^{35}\right) \theta\left(q^{5} ; q^{35}\right)-\theta\left(q^{24} ; q^{35}\right) \theta\left(q^{6} ; q^{35}\right) \theta\left(q^{27} ; q^{35}\right) \theta\left(q ; q^{35}\right) \\
=q \theta\left(q^{2} ; q^{35}\right) \theta\left(q^{4} ; q^{35}\right) \theta\left(q^{29} ; q^{35}\right) \theta\left(q ; q^{35}\right)
\end{array}
$$

\footnotetext{
${ }^{2}$ In case the reader wonders how we came up with these choices of $u, v, x, y$ (and the choices in subsequent proofs): after a lot of trial and error which produced some useful choices in certain cases, but did not lead to the recognition of any underlying patterns (we doubt in fact that there are), we decided to write a Maple programme that goes through all possible choices of $u, v, x, y$ in non-negative powers of $q$ and outputs choices that are appropriate to establish our identities.
} 
and thus the above right-hand side becomes

$$
\frac{\theta\left(q^{28} ; q^{35}\right) \theta\left(q^{2} ; q^{35}\right) \theta\left(q^{23} ; q^{35}\right) \theta\left(q^{5} ; q^{35}\right)\left(q^{35} ; q^{35}\right)_{\infty}}{\theta\left(q^{4} ; q^{35}\right) \theta\left(q^{11} ; q^{35}\right) \theta\left(q^{6} ; q^{35}\right) \theta\left(q ; q^{35}\right)},
$$

which is equivalent to the right-hand side of (2.3).

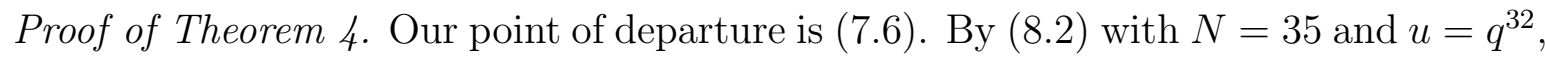
respectively with $N=35$ and $u=q^{18}$, we get

$$
\sum_{n=0}^{\infty}(-1)^{t(n)} q^{a_{n}}=\frac{\left(q^{29}, q^{6}, q^{35} ; q^{35}\right)_{\infty}}{\left(q^{32}, q^{3} ; q^{35}\right)_{\infty}}-q^{5} \frac{\left(q, q^{34}, q^{35} ; q^{35}\right)_{\infty}}{\left(q^{18}, q^{17} ; q^{35}\right)_{\infty}} .
$$

If we now replace $q$ by $q^{35}$ and choose $u=q^{7}, v=q^{15}, x=q$, and $y=q^{2}$ in (8.1), we obtain

$$
\begin{array}{r}
\theta\left(q^{17} ; q^{35}\right) \theta\left(q^{8} ; q^{35}\right) \theta\left(q^{6} ; q^{35}\right) \theta\left(q^{13} ; q^{35}\right)-\theta\left(q^{9} ; q^{35}\right) \theta\left(q^{16} ; q^{35}\right) \theta\left(q^{14} ; q^{35}\right) \theta\left(q^{5} ; q^{35}\right) \\
=q^{5} \theta\left(q ; q^{35}\right) \theta\left(q^{8} ; q^{35}\right) \theta\left(q^{22} ; q^{35}\right) \theta\left(q^{3} ; q^{35}\right)
\end{array}
$$

after little manipulation. Thus, the above right-hand side becomes

$$
\frac{\theta\left(q^{9} ; q^{35}\right) \theta\left(q^{16} ; q^{35}\right) \theta\left(q^{14} ; q^{35}\right) \theta\left(q^{5} ; q^{35}\right)\left(q^{35} ; q^{35}\right)_{\infty}}{\theta\left(q^{17} ; q^{35}\right) \theta\left(q^{3} ; q^{35}\right) \theta\left(q^{13} ; q^{35}\right) \theta\left(q^{8} ; q^{35}\right)},
$$

which is equivalent to the right-hand side of (2.4).

Proof of Theorem [5. Our point of departure is (7.7). By (8.2) with $N=35$ and $u=q^{29}$, respectively with $N=35$ and $u=q^{34}$, we get

$$
\sum_{n=0}^{\infty}(-1)^{\lfloor(n+4) / 8\rfloor} q^{a_{n}}=\frac{\left(q^{23}, q^{12}, q^{35} ; q^{35}\right)_{\infty}}{\left(q^{29}, q^{6} ; q^{35}\right)_{\infty}}+q \frac{\left(q^{33}, q^{2}, q^{35} ; q^{35}\right)_{\infty}}{\left(q^{34}, q ; q^{35}\right)_{\infty}} .
$$

If we now replace $q$ by $q^{35}$ and choose $u=q^{5}, v=q^{14}, x=q^{2}$, and $y=q^{4}$ in (8.1), we obtain

$$
\begin{array}{r}
\theta\left(q^{18} ; q^{35}\right) \theta\left(q^{3} ; q^{35}\right) \theta\left(q^{10} ; q^{35}\right) \theta\left(q^{7} ; q^{35}\right)-\theta\left(q^{12} ; q^{35}\right) \theta\left(q^{9} ; q^{35}\right) \theta\left(q^{16} ; q^{35}\right) \theta\left(q ; q^{35}\right) \\
=q \theta\left(q^{9} ; q^{35}\right) \theta\left(q^{6} ; q^{35}\right) \theta\left(q^{19} ; q^{35}\right) \theta\left(q^{2} ; q^{35}\right)
\end{array}
$$

after little manipulation. Thus, the above right-hand side becomes

$$
\frac{\theta\left(q^{18} ; q^{35}\right) \theta\left(q^{3} ; q^{35}\right) \theta\left(q^{10} ; q^{35}\right) \theta\left(q^{7} ; q^{35}\right)\left(q^{35} ; q^{35}\right)_{\infty}}{\theta\left(q^{6} ; q^{35}\right) \theta\left(q ; q^{35}\right) \theta\left(q^{16} ; q^{35}\right) \theta\left(q^{9} ; q^{35}\right)},
$$

which is equivalent to the right-hand side of (2.5).

Proof of Theorem 6. Our point of departure is (7.8). By (8.2) with $N=35$ and $u=q^{27}$, respectively with $N=35$ and $u=q^{22}$, we get

$$
\sum_{n=0}^{\infty}(-1)^{t(n)} q^{a_{n}}=\frac{\left(q^{19}, q^{16}, q^{35} ; q^{35}\right)_{\infty}}{\left(q^{27}, q^{8} ; q^{35}\right)_{\infty}}+q^{2} \frac{\left(q^{9}, q^{26}, q^{35} ; q^{35}\right)_{\infty}}{\left(q^{22}, q^{13} ; q^{35}\right)_{\infty}} .
$$

If we now replace $q$ by $q^{35}$ and choose $u=q^{7}, v=q^{16}, x=q^{3}$, and $y=q^{5}$ in (8.1), we obtain

$$
\begin{array}{r}
\theta\left(q^{21} ; q^{35}\right) \theta\left(q^{4} ; q^{35}\right) \theta\left(q^{11} ; q^{35}\right) \theta\left(q^{10} ; q^{35}\right)- \\
=\left(q^{13} ; q^{35}\right) \theta\left(q^{12} ; q^{35}\right) \theta\left(q^{19} ; q^{35}\right) \theta\left(q^{2} ; q^{35}\right) \\
=q^{2} \theta\left(q^{9} ; q^{35}\right) \theta\left(q^{8} ; q^{35}\right) \theta\left(q^{23} ; q^{35}\right) \theta\left(q^{2} ; q^{35}\right)
\end{array}
$$


after little manipulation. Thus, the above right-hand side becomes

$$
\frac{\theta\left(q^{21} ; q^{35}\right) \theta\left(q^{4} ; q^{35}\right) \theta\left(q^{11} ; q^{35}\right) \theta\left(q^{10} ; q^{35}\right)\left(q^{35} ; q^{35}\right)_{\infty}}{\theta\left(q^{8} ; q^{35}\right) \theta\left(q^{13} ; q^{35}\right) \theta\left(q^{12} ; q^{35}\right) \theta\left(q^{2} ; q^{35}\right)}
$$

which is equivalent to the right-hand side of (2.6) $)$.

Proof of Theorem 7. Our point of departure is (7.9). By (8.2) with $N=40$ and $u=$ $-q^{33}$, respectively with $N=40$ and $u=-q^{23}$, we get

$$
\sum_{n=0}^{\infty}(-1)^{\lfloor(n+2) / 4\rfloor} q^{a_{n}}=\frac{\left(q^{26}, q^{14}, q^{40} ; q^{40}\right)_{\infty}}{\left(-q^{33},-q^{7} ; q^{40}\right)_{\infty}}+q^{4} \frac{\left(q^{6}, q^{34}, q^{40} ; q^{40}\right)_{\infty}}{\left(-q^{23},-q^{17} ; q^{40}\right)_{\infty}} .
$$

If we now replace $q$ by $q^{40}$ and choose $u=-q^{9}, v=q^{16}, x=-q$, and $y=-q^{5}$ in (8.1), we obtain

$$
\begin{aligned}
& \theta\left(-q^{21} ; q^{40}\right) \theta\left(q^{8} ; q^{40}\right) \theta\left(q^{10} ; q^{40}\right) \theta\left(-q^{11} ; q^{40}\right) \\
& -\theta\left(q^{14} ; q^{40}\right) \theta\left(-q^{15} ; q^{40}\right) \theta\left(-q^{17} ; q^{40}\right) \theta\left(q^{4} ; q^{40}\right) \\
& \quad=q^{4} \theta\left(q^{6} ; q^{40}\right) \theta\left(-q^{7} ; q^{40}\right) \theta\left(-q^{25} ; q^{40}\right) \theta\left(q^{4} ; q^{40}\right)
\end{aligned}
$$

after little manipulation. Thus, the above right-hand side becomes

$$
\frac{\theta\left(-q^{21} ; q^{40}\right) \theta\left(q^{8} ; q^{40}\right) \theta\left(q^{10} ; q^{40}\right) \theta\left(-q^{11} ; q^{40}\right) \theta\left(q ; q^{40}\right)\left(q^{40} ; q^{40}\right)_{\infty}}{\theta\left(-q^{17} ; q^{40}\right) \theta\left(-q^{7} ; q^{40}\right) \theta\left(-q^{15} ; q^{40}\right) \theta\left(q^{4} ; q^{40}\right)}
$$

which is equivalent to the right-hand side of (2.7).

Proof of Theorem 8. Our point of departure is the first two lines in (7.10). By (8.2) with $N=40$ and $u=-q^{31}$, respectively with $N=40$ and $u=-q^{39}$, we get

$$
\sum_{n=0}^{\infty}(-1)^{\lfloor 5 n / 4\rfloor} q^{a_{n}}=\frac{\left(q^{22}, q^{18}, q^{40} ; q^{40}\right)_{\infty}}{\left(-q^{31},-q^{9} ; q^{40}\right)_{\infty}}-q \frac{\left(q^{38}, q^{2}, q^{40} ; q^{40}\right)_{\infty}}{\left(-q^{39},-q ; q^{40}\right)_{\infty}} .
$$

If we now replace $q$ by $q^{40}$ and choose $u=-q^{8}, v=q^{5}, x=q^{17}$, and $y=q^{7}$ in (8.1), we obtain

$$
\begin{aligned}
\theta\left(q^{24} ; q^{40}\right) \theta\left(q^{10} ; q^{40}\right) & \theta\left(-q^{13} ; q^{40}\right) \theta\left(-q^{3} ; q^{40}\right) \\
-\theta\left(q^{22} ; q^{40}\right) \theta\left(q^{12} ; q^{40}\right) \theta\left(-q^{15} ; q^{40}\right) \theta\left(-q ; q^{40}\right) & \\
\quad & =-q \theta\left(q^{12} ; q^{40}\right) \theta\left(q^{2} ; q^{40}\right) \theta\left(-q^{25} ; q^{40}\right) \theta\left(-q^{9} ; q^{40}\right),
\end{aligned}
$$

and thus the above right-hand side becomes

$$
\frac{\theta\left(q^{24} ; q^{40}\right) \theta\left(q^{10} ; q^{40}\right) \theta\left(-q^{13} ; q^{40}\right) \theta\left(-q^{3} ; q^{40}\right)\left(q^{40} ; q^{40}\right)_{\infty}}{\theta\left(-q ; q^{40}\right) \theta\left(-q^{9} ; q^{40}\right) \theta\left(q^{12} ; q^{40}\right) \theta\left(-q^{15} ; q^{40}\right)},
$$

which is equivalent to the right-hand side of (2.8).

Proof of Theorem 9. The point of departure is the first two lines in (7.11). By (8.2) with $N=40$ and $u=-q^{37}$, respectively with $N=40$ and $u=-q^{27}$, we get

$$
\sum_{n=0}^{\infty}(-1)^{\lfloor(n+2) / 4\rfloor} q^{a_{n}}=\frac{\left(q^{34}, q^{6}, q^{40} ; q^{40}\right)_{\infty}}{\left(-q^{37},-q^{3} ; q^{40}\right)_{\infty}}+q \frac{\left(q^{14}, q^{26}, q^{40} ; q^{40}\right)_{\infty}}{\left(-q^{27},-q^{13} ; q^{40}\right)_{\infty}} .
$$


By (8.1) with $q$ replaced by $q^{40}, u=-q^{16}, v=q^{11}, y=q^{15}$, and $x=q^{19}$, we get

$$
\begin{array}{r}
\theta\left(q^{34} ; q^{40}\right) \theta\left(q^{4} ; q^{40}\right) \theta\left(-q^{27} ; q^{40}\right) \theta\left(-q^{5} ; q^{40}\right)-\theta\left(q^{30} ; q^{40}\right) \theta\left(q^{8} ; q^{40}\right) \theta\left(-q^{31} ; q^{40}\right) \theta\left(-q ; q^{40}\right) \\
=-q \theta\left(q^{26} ; q^{40}\right) \theta\left(q^{4} ; q^{40}\right) \theta\left(-q^{35} ; q^{40}\right) \theta\left(-q^{3} ; q^{40}\right)
\end{array}
$$

and thus the above right-hand side becomes

$$
\frac{\theta\left(q^{30} ; q^{40}\right) \theta\left(q^{8} ; q^{40}\right) \theta\left(-q^{31} ; q^{40}\right) \theta\left(-q ; q^{40}\right)\left(q^{40} ; q^{40}\right)_{\infty}}{\theta\left(-q^{27} ; q^{40}\right) \theta\left(-q^{37} ; q^{40}\right) \theta\left(q^{4} ; q^{40}\right) \theta\left(-q^{5} ; q^{40}\right)}
$$

which is equivalent to the right-hand side of (2.9).

Proof of Theorem 10. Our point of departure is the first two lines in (7.12). By (8.2) with $N=40$ and $u=-q^{29}$, respectively by (8.3) with $N=40$ and $u=-q^{59}$, we get

$$
\sum_{n=0}^{\infty}(-1)^{\lfloor 5 n / 4\rfloor} q^{a_{n}}=\frac{\left(q^{18}, q^{22}, q^{40} ; q^{40}\right)_{\infty}}{\left(-q^{29},-q^{11} ; q^{40}\right)_{\infty}}-q^{5} \frac{\left(q^{38}, q^{2}, q^{40} ; q^{40}\right)_{\infty}}{\left(-q^{19},-q^{21} ; q^{40}\right)_{\infty}}
$$

If we now replace $q$ by $q^{40}$ and choose $u=-q^{13}, v=-q^{15}, x=-q^{3}$, and $y=q^{8}$ in (8.1), we obtain

$$
\begin{aligned}
\theta\left(-q^{23} ; q^{40}\right) \theta\left(q^{10} ; q^{40}\right) \theta\left(q^{16} ; q^{40}\right) \theta\left(-q^{7} ; q^{40}\right) & \\
-\theta\left(-q^{21} ; q^{40}\right) & \theta\left(q^{12} ; q^{40}\right) \theta\left(q^{18} ; q^{40}\right) \theta\left(-q^{5} ; q^{40}\right) \\
& =-q^{5} \theta\left(-q^{11} ; q^{40}\right) \theta\left(q^{2} ; q^{40}\right) \theta\left(q^{28} ; q^{40}\right) \theta\left(-q^{5} ; q^{40}\right)
\end{aligned}
$$

after little manipulation. Thus, the above right-hand side becomes

$$
\frac{\theta\left(-q^{23} ; q^{40}\right) \theta\left(q^{10} ; q^{40}\right) \theta\left(q^{16} ; q^{40}\right) \theta\left(-q^{7} ; q^{40}\right)\left(q^{40} ; q^{40}\right)_{\infty}}{\theta\left(-q^{11} ; q^{40}\right) \theta\left(-q^{19} ; q^{40}\right) \theta\left(q^{12} ; q^{40}\right) \theta\left(-q^{5} ; q^{40}\right)},
$$

which is equivalent to the right-hand side of (2.10).

Proof of Theorem 11. This is a special case of Theorem 36.

Proof of Theorem 12. This is a special case of Theorem 36.

Proof of Theorem 13. As we already said, this is a direct consequence of the Jacobi triple product identity (4.1): one replaces $q$ by $q^{10}$ and then chooses $z=-q^{6}$ there.

Proof of Theorem 14. We start again with (cf. (17.13))

$$
\sum_{n=0}^{\infty}\left(q^{n(n+1)}-q^{5 n(n+1)+1}\right)=\left(q^{2} ; q^{2}\right)_{\infty}\left(-q^{2} ; q^{2}\right)_{\infty}^{2}-q\left(q^{10} ; q^{10}\right)_{\infty}\left(-q^{10} ; q^{10}\right)_{\infty}^{2}
$$

The right-hand side can be rewritten as

$$
\frac{\left(q^{4} ; q^{4}\right)_{\infty}^{2}}{\left(q^{2} ; q^{2}\right)_{\infty}}-q \frac{\left(q^{20} ; q^{20}\right)_{\infty}^{2}}{\left.q^{10} ; q^{10}\right)_{\infty}}=\frac{\left(q^{20} ; q^{20}\right)_{\infty}^{2}}{\left.q^{10} ; q^{10}\right)_{\infty}}\left(\frac{\theta\left(q^{4} ; q^{20}\right) \theta\left(q^{8} ; q^{20}\right)}{\theta\left(q^{2} ; q^{20}\right) \theta\left(q^{6} ; q^{20}\right)}-q\right)
$$

If in (8.1) we replace $q$ by $q^{20}$ and choose $u=q^{5}, v=q^{2}, x=q^{12}$, and $y=q^{4}$, then we obtain

$$
\begin{array}{r}
\theta\left(q^{16} ; q^{20}\right) \theta\left(q^{8} ; q^{20}\right) \theta\left(q^{7} ; q^{20}\right) \theta\left(q^{3} ; q^{20}\right)-\theta\left(q^{14} ; q^{20}\right) \theta\left(q^{10} ; q^{20}\right) \theta\left(q^{9} ; q^{20}\right) \theta\left(q ; q^{20}\right) \\
=q \theta\left(q^{6} ; q^{20}\right) \theta\left(q^{2} ; q^{20}\right) \theta\left(q^{17} ; q^{20}\right) \theta\left(q^{7} ; q^{20}\right) .
\end{array}
$$


If this is used in (9.1), then we obtain the right-hand side of (2.14) after little manipulation.

Proof of Theorem 15. We start again with (cf. (7.14))

$$
1+\sum_{n=1}^{\infty}\left(q^{n^{2}}+q^{5 n^{2}}\right)=\frac{1}{2}\left(\left(q^{2} ; q^{2}\right)_{\infty}\left(-q ; q^{2}\right)_{\infty}^{2}+\left(q^{10} ; q^{10}\right)_{\infty}\left(-q^{5} ; q^{10}\right)_{\infty}^{2}\right)
$$

Now, we have the relation' 3 (cf. [2, Eq. (8.10.9)])

$$
\left(q ; q^{2}\right)_{\infty}^{-1}=(-q ; q)_{\infty}
$$

Upon replacement of $q$ by $-q$, we obtain the variant

$$
\left(-q ; q^{2}\right)_{\infty}^{-1}=\left(q ; q^{2}\right)_{\infty}\left(-q^{2} ; q^{2}\right)_{\infty}
$$

We use the latter identity in (9.2) and get

$$
\begin{array}{r}
1+\sum_{n=1}^{\infty}\left(q^{n^{2}}+q^{5 n^{2}}\right)=\frac{1}{2}\left(\frac{\left(q^{2} ; q^{2}\right)_{\infty}\left(-q ; q^{2}\right)_{\infty}}{\left(q ; q^{2}\right)_{\infty}\left(-q^{2} ; q^{2}\right)_{\infty}}+\left(q^{10} ; q^{10}\right)_{\infty}\left(-q^{5} ; q^{10}\right)_{\infty}^{2}\right) \\
=\frac{1}{2}\left(\frac{\theta\left(q^{2} ; q^{10}\right) \theta\left(q^{4} ; q^{10}\right)\left(q^{10} ; q^{10}\right)_{\infty} \theta\left(-q ; q^{10}\right) \theta\left(-q^{3} ; q^{10}\right)\left(-q^{5} ; q^{10}\right)_{\infty}}{\theta\left(q ; q^{10}\right) \theta\left(q^{3} ; q^{10}\right)\left(q^{5} ; q^{10}\right)_{\infty} \theta\left(-q^{2} ; q^{10}\right) \theta\left(-q^{4} ; q^{10}\right)\left(-q^{10} ; q^{10}\right)_{\infty}}\right. \\
\left.+\left(q^{10} ; q^{10}\right)_{\infty}\left(-q^{5} ; q^{10}\right)_{\infty}^{2}\right)
\end{array}
$$

If, in the first expression within parentheses we use (9.4) with $q$ replaced by $q^{5}$, then the above becomes

$$
\begin{aligned}
1+\sum_{n=1}^{\infty}\left(q^{n^{2}}+q^{5 n^{2}}\right) & =\frac{\left(q^{10} ; q^{10}\right)_{\infty}\left(-q^{5} ; q^{10}\right)_{\infty}^{2}}{2 \theta\left(q ; q^{10}\right) \theta\left(q^{3} ; q^{10}\right) \theta\left(-q^{2} ; q^{10}\right) \theta\left(-q^{4} ; q^{10}\right)} \\
& \times\left(\theta\left(q^{2} ; q^{10}\right) \theta\left(q^{4} ; q^{10}\right) \theta\left(-q ; q^{10}\right) \theta\left(-q^{3} ; q^{10}\right)\right. \\
& \left.\quad+\theta\left(q ; q^{10}\right) \theta\left(q^{3} ; q^{10}\right) \theta\left(-q^{2} ; q^{10}\right) \theta\left(-q^{4} ; q^{10}\right)\right) .
\end{aligned}
$$

We now replace $q$ by $q^{10}$ in (8.1) and choose $u=-q^{3}, v=q, x=q^{4}$, and $y=q^{3}$. This gives the relation

$$
\begin{aligned}
\theta\left(q^{2} ; q^{10}\right) \theta\left(q^{4} ; q^{10}\right) \theta\left(-q ; q^{10}\right) \theta\left(-q^{3} ; q^{10}\right) & +\theta\left(q ; q^{10}\right) \theta\left(q^{3} ; q^{10}\right) \theta\left(-q^{2} ; q^{10}\right) \theta\left(-q^{4} ; q^{10}\right) \\
& =\theta\left(q^{3} ; q^{10}\right) \theta\left(-q^{4} ; q^{10}\right) \theta\left(q^{5} ; q^{10}\right) \theta\left(-1 ; q^{10}\right) .
\end{aligned}
$$

If this is used in the above expression, then we obtain (2.15) after some manipulation, where (9.4) is again used with $q$ replaced by $q^{5}$.

Proof of Theorem 16. As we already said, this is a direct consequence of the Jacobi triple product identity (4.1): one replaces $q$ by $q^{10}$ and then chooses $z=-q^{7}$ there.

\footnotetext{
${ }^{3}$ In combinatorial terms, this is Euler's theorem that the number of partitions of $n$ into odd parts equals the number of partitions of $n$ into distinct parts.
} 
Proof of Theorem 17. We start again with (cf. (17.15))

$$
\sum_{n=0}^{\infty}\left(q^{n(n+1)}+q^{5 n(n+1)+1}\right)=\left(q^{2} ; q^{2}\right)_{\infty}\left(-q^{2} ; q^{2}\right)_{\infty}^{2}+q\left(q^{10} ; q^{10}\right)_{\infty}\left(-q^{10} ; q^{10}\right)_{\infty}^{2}
$$

The right-hand side can be rewritten as

$$
\frac{\left(q^{4} ; q^{4}\right)_{\infty}^{2}}{\left(q^{2} ; q^{2}\right)_{\infty}}+q \frac{\left(q^{20} ; q^{20}\right)_{\infty}^{2}}{\left(q^{10} ; q^{10}\right)_{\infty}}=\frac{\left(q^{20} ; q^{20}\right)_{\infty}^{2}}{\left(q^{10} ; q^{10}\right)_{\infty}}\left(\frac{\theta\left(q^{4} ; q^{20}\right) \theta\left(q^{8} ; q^{20}\right)}{\theta\left(q^{2} ; q^{20}\right) \theta\left(q^{6} ; q^{20}\right)}+q\right)
$$

If in (8.1) we replace $q$ by $q^{20}$ and choose $u=q^{5}, v=q^{2}, x=q^{6}$, and $y=q^{4}$, then we obtain

$$
\begin{aligned}
\theta\left(q^{10} ; q^{20}\right) \theta\left(q^{2} ; q^{20}\right) \theta\left(q^{7} ; q^{20}\right) \theta\left(q^{3} ; q^{20}\right)-\theta & \left(q^{8} ; q^{20}\right) \theta\left(q^{4} ; q^{20}\right) \theta\left(q^{9} ; q^{20}\right) \theta\left(q ; q^{20}\right) \\
& =q \theta\left(q^{6} ; q^{20}\right) \theta\left(q^{2} ; q^{20}\right) \theta\left(q^{11} ; q^{20}\right) \theta\left(q ; q^{20}\right) .
\end{aligned}
$$

If this is used in (9.5), then we obtain the right-hand side of (2.17) after little manipulation.

Proof of Theorem 18. As we already said, this is a direct consequence of the Jacobi triple product identity (4.1): one replaces $q$ by $q^{10}$ and then chooses $z=-q^{8}$ there.

Proof of Theorem 19. As we already said, this is a direct consequence of the Jacobi triple product identity (4.1): one replaces $q$ by $q^{10}$ and then chooses $z=-q^{9}$ there.

Proof of Theorem 20. We start again with (cf. (7.16))

$$
\sum_{n=1}^{\infty}\left(q^{n^{2}-1}-q^{5 n^{2}-1}\right)=\frac{1}{2 q}\left(\left(q^{2} ; q^{2}\right)_{\infty}\left(-q ; q^{2}\right)_{\infty}^{2}-\left(q^{10} ; q^{10}\right)_{\infty}\left(-q^{5} ; q^{10}\right)_{\infty}^{2}\right)
$$

The right-hand side is almost the same expression as the one on the right-hand side of (9.2), except for a multiplicative factor of $1 / q$ and a changed sign. Hence, by proceeding as in the alternative proof of Theorem 15, we arrive at

$$
\begin{aligned}
\sum_{n=1}^{\infty}\left(q^{n^{2}-1}-q^{5 n^{2}-1}\right) & =\frac{\left(q^{10} ; q^{10}\right)_{\infty}\left(-q^{5} ; q^{10}\right)_{\infty}^{2}}{2 q \theta\left(q ; q^{10}\right) \theta\left(q^{3} ; q^{10}\right) \theta\left(-q^{2} ; q^{10}\right) \theta\left(-q^{4} ; q^{10}\right)} \\
\times\left(\theta\left(q^{2} ; q^{10}\right) \theta\left(q^{4} ; q^{10}\right) \theta\left(-q ; q^{10}\right) \theta\left(-q^{3} ; q^{10}\right)\right. & \left.-\theta\left(q ; q^{10}\right) \theta\left(q^{3} ; q^{10}\right) \theta\left(-q^{2} ; q^{10}\right) \theta\left(-q^{4} ; q^{10}\right)\right) .
\end{aligned}
$$

We now replace $q$ by $q^{10}$ in (8.1) and choose $u=-q^{3}, v=q, x=q^{4}$, and $y=q^{3}$. This gives the relation

$$
\begin{aligned}
\theta\left(q^{4} ; q^{10}\right) \theta\left(q^{2} ; q^{10}\right) \theta\left(-q^{3} ; q^{10}\right) \theta\left(-q ; q^{10}\right) & -\theta\left(-q^{4} ; q^{10}\right) \theta\left(-q^{2} ; q^{10}\right) \theta\left(q^{3} ; q^{10}\right) \theta\left(q ; q^{10}\right) \\
& =q \theta\left(-q^{2} ; q^{10}\right) \theta\left(-1 ; q^{10}\right) \theta\left(q^{5} ; q^{10}\right) \theta\left(q ; q^{10}\right) .
\end{aligned}
$$

If this is used in the above expression, then we obtain (2.20) after some manipulation, where (9.4) is again used with $q$ replaced by $q^{5}$.

Proof of Theorem 21. This is a special case of Corollary 35.

Proof of Theorem 22. This theorem is a special case of Theorem 34.

Proof of Theorem 23. This is a special case of Corollary 35. 
Proof of Theorem 24. This theorem is a special case of Theorem 34.

Proof of Theorem 25. This theorem is a special case of Theorem 34.

Proof of Theorem 26. Using (8.2) with $N=8$ and $u=q^{7}$, the right-hand side of (7.17) can be simplified into one product. This gives the claimed result.

Proof of Theorem 27. Using (8.3) with $N=8$ and $u=q^{11}$, the right-hand side of (7.18) can be simplified into one product. This gives the claimed result.

Proof of Theorem 28. This theorem is a special case of Theorem 36.

Proof of Theorem 29, This theorem is a special case of Theorem 36.

Proof of Theorem 30. This theorem is a special case of Corollary 37.

Proof of Theorem 31. This is a special case of Theorem 38.

Proof of Theorem 32. This is a special case of Theorem 38.

\section{Parametric FAmilies of Generating FUnCtions For SEQUenCes of SQUARES}

Here we present two results on generating functions for sequences of squares that contain parameters. The first of these results consists in Theorem 34 and Corollary 35 , while the second consists in Theorem 36 and Corollary 37 . For each theorem-corollary pair the proof is the same, but the theorem covers a parameter range that is different from that of the corollary. We conclude the section by stating, and proving, a uniform version of Theorems 31 and 32 .

The following theorem covers Theorems 22, 24] and 25,

Theorem 34. Let $P$ be an odd prime power different from a power of 3 , and let a be an odd positive integer relatively prime to $P$ and less than $P$. Let $\left(a_{n}\right)_{n \geq 0}$ be the sequence of non-negative integers $m$ such that $24 P m+a^{2}$ is a square.

(1) If $a \equiv P(\bmod 3)$, then

$$
\sum_{n=0}^{\infty}(-1)^{\lfloor(n+2) / 4\rfloor} q^{a_{n}}=\frac{\left(q^{(P-a) / 3}, q^{(2 P+a) / 3}, q^{P} ; q^{P}\right)_{\infty}}{\left(q^{(P-a) / 6}, q^{(5 P+a) / 6} ; q^{P}\right)_{\infty}} .
$$

(2) If $a \not \equiv P(\bmod 3)$, then

$$
\sum_{n=0}^{\infty}(-1)^{\lfloor(n+2) / 4\rfloor} q^{a_{n}}=\frac{\left(q^{(P+a) / 3}, q^{(2 P-a) / 3}, q^{P} ; q^{P}\right)_{\infty}}{\left(q^{(P+a) / 6}, q^{(5 P-a) / 6} ; q^{P}\right)_{\infty}} .
$$

Proof. We have to find all $S$ such that

$$
S^{2} \equiv a^{2} \quad(\bmod 24 P) .
$$

We claim that there are the following two cases:

(C1) If $a \equiv P(\bmod 3)$, then $S \equiv a, 2 P-a, 4 P+a, 6 P-a(\bmod 6 P)$.

(C2) If $a \not \equiv P(\bmod 3)$, then $S \equiv a, 2 P+a, 4 P-a, 6 P-a(\bmod 6 P)$. 
It should be noted that the condition $a<P$ guarantees that, in both cases, the congruence classes above are listed in increasing order.

By assumption, $a$ is odd. Hence, also $S$ must be odd and automatically $S^{2} \equiv a^{2} \equiv$ $1(\bmod 8)$. Consequently, the congruence $(10.3)$ can be reduced to

$$
(S-a)(S+a) \equiv 0 \quad(\bmod 6 P) .
$$

Two solutions are immediate, namely $S \equiv a(\bmod 6 P)$ and $S \equiv-a(\bmod 6 P)$. There are two further possibilities:

$$
S \equiv a(\bmod 6) \quad \text { and } \quad S \equiv-a(\bmod P),
$$

and

$$
S \equiv-a(\bmod 6) \text { and } S \equiv a(\bmod P) .
$$

Under our assumption that $a$ is relatively prime to $P$, there are no other possibilities. For, writing $P=p^{e}$ with $p$ an odd prime number, the simultaneous congruences

$$
S \equiv a\left(\bmod p^{\alpha}\right) \text { and } S \equiv-a\left(\bmod p^{\beta}\right)
$$

with $\alpha+\beta=e$ and both $\alpha$ and $\beta$ positive would imply that $2 a$ is divisible by $p$, a contradiction to our assumptions.

It is straightforward to see that, depending on whether $a \equiv P(\bmod 3)$ or not, the solutions to (10.4) respectively to (10.5) are given by the second and third option in (C1) and (C2) above.

We now discuss Case (C1). Here, we have

$$
\begin{gathered}
\sum_{n=0}^{\infty}(-1)^{\lfloor(n+2) / 4\rfloor} q^{a_{n}}=\sum_{k=0}^{\infty}(-1)^{k} q^{\frac{1}{24 P}\left((6 P k+a)^{2}-a^{2}\right)}+\sum_{k=0}^{\infty}(-1)^{k} q^{\frac{1}{24 P}\left((6 P k+2 P-a)^{2}-a^{2}\right)} \\
-\sum_{k=0}^{\infty}(-1)^{k} q^{\frac{1}{24 P}\left((6 P k+4 P+a)^{2}-a^{2}\right)}-\sum_{k=0}^{\infty}(-1)^{k} q^{\frac{1}{24 P}\left((6 P k+6 P-a)^{2}-a^{2}\right)} \\
=\sum_{k=0}^{\infty}(-1)^{k} q^{\frac{1}{2}\left(3 P k^{2}+a k\right)}+\sum_{k=0}^{\infty}(-1)^{k} q^{\frac{1}{2}\left(3 P k^{2}+(2 P-a) k\right)+\frac{1}{6}(P-a)} \\
\quad-\sum_{k=0}^{\infty}(-1)^{k} q^{\frac{1}{2}\left(3 P k^{2}+(4 P+a) k\right)+\frac{1}{3}(2 P+a)}-\sum_{k=0}^{\infty}(-1)^{k} q^{\frac{1}{2}\left(3 P k^{2}+(6 P-a) k\right)+\frac{1}{2}(3 P-a)} .
\end{gathered}
$$

Again, sums can be put together in pairs, so that one obtains two sums over all integers $k$ :

$$
\sum_{n=0}^{\infty}(-1)^{\lfloor(n+2) / 4\rfloor} q^{a_{n}}=\sum_{k=-\infty}^{\infty}(-1)^{k} q^{\frac{1}{2}\left(3 P k^{2}+a k\right)}+\sum_{k=-\infty}^{\infty}(-1)^{k} q^{\frac{1}{2}\left(3 P k^{2}+(2 P-a) k\right)+\frac{1}{6}(P-a)} .
$$

Now, to each of these sums we apply the Jacobi triple product identity (4.1) to get

$$
\begin{aligned}
\sum_{n=0}^{\infty}(-1)^{\lfloor(n+2) / 4\rfloor} q^{a_{n}}=\left(q^{3 P}, q^{(3 P+a) / 2}, q^{(3 P-a) / 2} ; q^{3 P}\right)_{\infty} & \\
& +q^{(P-a) / 6}\left(q^{3 P}, q^{(5 P-a) / 2}, q^{(P+a) / 2} ; q^{3 P}\right)_{\infty} .
\end{aligned}
$$

The sum of these two products simplifies to the single product on the right-hand side of (10.1) as is seen by applying (8.2) with $N=P$ and $u=q^{(5 P+a) / 6}$. 
On the other hand, if we are in Case (C2), then we have

$$
\begin{gathered}
\sum_{n=0}^{\infty}(-1)^{\lfloor(n+2) / 4\rfloor} q^{a_{n}}=\sum_{k=0}^{\infty}(-1)^{k} q^{\frac{1}{24 P}\left((6 P k+a)^{2}-a^{2}\right)}+\sum_{k=0}^{\infty}(-1)^{k} q^{\frac{1}{24 P}\left((6 P k+2 P+a)^{2}-a^{2}\right)} \\
\quad-\sum_{k=0}^{\infty}(-1)^{k} q^{\frac{1}{24 P}\left((6 P k+4 P-a)^{2}-a^{2}\right)}-\sum_{k=0}^{\infty}(-1)^{k} q^{\frac{1}{24 P}\left((6 P k+6 P-a)^{2}-a^{2}\right)} \\
=\sum_{k=0}^{\infty}(-1)^{k} q^{\frac{1}{2}\left(3 P k^{2}+a k\right)}+\sum_{k=0}^{\infty}(-1)^{k} q^{\frac{1}{2}\left(3 P k^{2}+(2 P+a) k\right)+\frac{1}{6}(P+a)} \\
\quad-\sum_{k=0}^{\infty}(-1)^{k} q^{\frac{1}{2}\left(3 P k^{2}+(4 P-a) k\right)+\frac{1}{3}(2 P-a)}-\sum_{k=0}^{\infty}(-1)^{k} q^{\frac{1}{2}\left(3 P k^{2}+(6 P-a) k\right)+\frac{1}{2}(3 P-a)} .
\end{gathered}
$$

Again, sums can be put together in pairs, so that one obtains two sums over all integers $k$ :

$$
\sum_{n=0}^{\infty}(-1)^{\lfloor(n+2) / 4\rfloor} q^{a_{n}}=\sum_{k=-\infty}^{\infty}(-1)^{k} q^{\frac{1}{2}\left(3 P k^{2}+a k\right)}+\sum_{k=-\infty}^{\infty}(-1)^{k} q^{\frac{1}{2}\left(3 P k^{2}+(2 P+a) k\right)+\frac{1}{6}(P+a)} .
$$

Now, to each of these sums we apply the Jacobi triple product identity (4.1) to get

$$
\begin{aligned}
\sum_{n=0}^{\infty}(-1)^{\lfloor(n+2) / 4\rfloor} q^{a_{n}}=\left(q^{3 P}, q^{(3 P+a) / 2}, q^{(3 P-a) / 2}\right. & \left.; q^{3 P}\right)_{\infty} \\
& +q^{(P+a) / 6}\left(q^{3 P}, q^{(5 P+a) / 2}, q^{(P-a) / 2} ; q^{3 P}\right)_{\infty} .
\end{aligned}
$$

The sum of these two products simplifies to the single product on the right-hand side of (10.2) as is seen by applying (8.2) with $N=P$ and $u=q^{(5 P-a) / 6}$.

The following corollary covers Theorems 21 and 23.

Corollary 35. Let $P$ be an odd prime power different from a power of 3, and let a be an odd positive integer relatively prime to $P$ with $P<a<2 P$. Let $\left(a_{n}\right)_{n \geq 0}$ be the sequence of non-negative integers $m$ such that $24 P m+a^{2}$ is a square.

(1) If $a \equiv P(\bmod 3)$, then

$$
\sum_{n=0}^{\infty}(-1)^{\lfloor(n+2) / 4\rfloor} q^{a_{n}}=\frac{\left(q^{(P-a) / 3}, q^{(2 P+a) / 3}, q^{P} ; q^{P}\right)_{\infty}}{\left(q^{(P-a) / 6}, q^{(5 P+a) / 6} ; q^{P}\right)_{\infty}}
$$

(2) If $a \not \equiv P(\bmod 3)$, then

$$
\sum_{n=0}^{\infty}(-1)^{\lfloor 5 n / 4\rfloor} q^{a_{n}}=\frac{\left(q^{(P+a) / 3}, q^{(2 P-a) / 3}, q^{P} ; q^{P}\right)_{\infty}}{\left(q^{(P+a) / 6}, q^{(5 P-a) / 6} ; q^{P}\right)_{\infty}}
$$

Proof. If one looks through the arguments of the proof of Theorem 34, then one sees that everything can be copied verbatim, until it comes to the description of the two cases to be considered: we have to adapt the order of the congruence classes, as shown below.

(C1') If $a \equiv P(\bmod 3)$, then $S \equiv 2 P-a, a, 6 P-a, 4 P+a(\bmod 6 P)$.

(C2') If $a \not \equiv P(\bmod 3)$, then $S \equiv a, 4 P-a, 2 P+a, 6 P-a(\bmod 6 P)$. 
While in Case (C1'), the rest of the proof can be copied, in Case (C2') this requires a change in sign in the original sum from $(-1)^{\lfloor(n+2) / 4\rfloor}$ to $(-1)^{\lfloor 5 n / 4\rfloor}$.

The following theorem covers Theorems 11, 12, 28 and 29.

Theorem 36. Let $P$ be an odd prime power different from a power of 3 , and let a be a positive integer relatively prime to $P$ and less than $P / 2$. Let $\left(a_{n}\right)_{n \geq 0}$ be the sequence of non-negative integers $m$ such that $3 P m+a^{2}$ is a square.

(1) If $a \equiv P(\bmod 3)$, then

$$
\sum_{n=0}^{\infty}(-1)^{\lfloor(n+2) / 4\rfloor} q^{a_{n}}=\frac{\left(q^{(4 P-4 a) / 3}, q^{(2 P+4 a) / 3}, q^{2 P} ; q^{2 P}\right)_{\infty}}{\left(q^{(5 P-2 a) / 3}, q^{(P+2 a) / 3} ; q^{2 P}\right)_{\infty}} .
$$

(2) If $a \not \equiv P(\bmod 3)$, then

$$
\sum_{n=0}^{\infty}(-1)^{\lfloor(n+2) / 4\rfloor} q^{a_{n}}=\frac{\left(q^{(4 P+4 a) / 3}, q^{(2 P-4 a) / 3}, q^{2 P} ; q^{2 P}\right)_{\infty}}{\left(q^{(5 P+2 a) / 3}, q^{(P-2 a) / 3} ; q^{2 P}\right)_{\infty}} .
$$

Proof. We have to find all $S$ such that

$$
S^{2} \equiv a^{2} \quad(\bmod 3 P) \text {. }
$$

As before, there are two cases:

(C1) If $a \equiv P(\bmod 3)$, then $S \equiv a, P+a, 2 P-a, 3 P-a(\bmod 3 P)$.

(C2) If $a \not \equiv P(\bmod 3)$, then $S \equiv a, P-a, 2 P+a, 3 P-a(\bmod 3 P)$.

We now discuss Case (C1). Here, we have

$$
\begin{gathered}
\sum_{n=0}^{\infty}(-1)^{\lfloor(n+2) / 4\rfloor} q^{a_{n}}=\sum_{k=0}^{\infty}(-1)^{k} q^{\frac{1}{3 P}\left((3 P k+a)^{2}-a^{2}\right)}+\sum_{k=0}^{\infty}(-1)^{k} q^{\frac{1}{3 P}\left((3 P k+P+a)^{2}-a^{2}\right)} \\
-\sum_{k=0}^{\infty}(-1)^{k} q^{\frac{1}{3 P}\left((3 P k+2 P-a)^{2}-a^{2}\right)}-\sum_{k=0}^{\infty}(-1)^{k} q^{\frac{1}{3 P}\left((3 P k+3 P-a)^{2}-a^{2}\right)} \\
=\sum_{k=0}^{\infty}(-1)^{k} q^{3 P k^{2}+2 a k}+\sum_{k=0}^{\infty}(-1)^{k} q^{3 P k^{2}+2(P+a) k+\frac{1}{3}(P+2 a)} \\
-\sum_{k=0}^{\infty}(-1)^{k} q^{3 P k^{2}+2(2 P-a) k+\frac{4}{3}(P-a)}-\sum_{k=0}^{\infty}(-1)^{k} q^{3 P k^{2}+2(3 P-a) k+3 P-2 a} .
\end{gathered}
$$

Again, sums can be put together in pairs, so that one obtains two sums over all integers $k$ :

$$
\sum_{n=0}^{\infty}(-1)^{\lfloor(n+2) / 4\rfloor} q^{a_{n}}=\sum_{k=-\infty}^{\infty}(-1)^{k} q^{3 P k^{2}+2 a k}+\sum_{k=-\infty}^{\infty}(-1)^{k} q^{3 P k^{2}+2(P+a) k+\frac{1}{3}(P+2 a)} .
$$

Now, to each of these sums we apply the Jacobi triple product identity (4.1) to get

$$
\begin{aligned}
\sum_{n=0}^{\infty}(-1)^{\lfloor(n+2) / 4\rfloor} q^{a_{n}}=\left(q^{6 P}, q^{3 P+2 a}, q^{3 P-2 a} ; q^{6 P}\right)_{\infty} & \\
& +q^{(P+2 a) / 3}\left(q^{6 P}, q^{5 P+2 a}, q^{P-2 a} ; q^{6 P}\right)_{\infty} .
\end{aligned}
$$


The sum of these two products simplifies to the single product on the right-hand side of (10.8) as is seen by applying (28.2) with $N=2 P$ and $u=q^{(5 P-2 a) / 3}$.

On the other hand, if we are in Case (C2), then we have

$$
\begin{gathered}
\sum_{n=0}^{\infty}(-1)^{\lfloor(n+2) / 4\rfloor} q^{a_{n}}=\sum_{k=0}^{\infty}(-1)^{k} q^{\frac{1}{3 P}\left((3 P k+a)^{2}-a^{2}\right)}+\sum_{k=0}^{\infty}(-1)^{k} q^{\frac{1}{3 P}\left((3 P k+P-a)^{2}-a^{2}\right)} \\
\quad-\sum_{k=0}^{\infty}(-1)^{k} q^{\frac{1}{3 P}\left((3 P k+2 P+a)^{2}-a^{2}\right)}-\sum_{k=0}^{\infty}(-1)^{k} q^{\frac{1}{3 P}\left((3 P k+3 P-a)^{2}-a^{2}\right)} \\
=\sum_{k=0}^{\infty}(-1)^{k} q^{3 P k^{2}+2 a k}+\sum_{k=0}^{\infty}(-1)^{k} q^{3 P k^{2}+2(P-a) k+\frac{1}{3}(P-2 a)} \\
-\sum_{k=0}^{\infty}(-1)^{k} q^{3 P k^{2}+2(2 P+a) k+\frac{4}{3}(P+a)}-\sum_{k=0}^{\infty}(-1)^{k} q^{3 P k^{2}+2(3 P-a) k+3 P-2 a} .
\end{gathered}
$$

Again, sums can be put together in pairs, so that one obtains two sums over all integers $k$ :

$$
\sum_{n=0}^{\infty}(-1)^{\lfloor(n+2) / 4\rfloor} q^{a_{n}}=\sum_{k=-\infty}^{\infty}(-1)^{k} q^{3 P k^{2}+2 a k}+\sum_{k=-\infty}^{\infty}(-1)^{k} q^{3 P k^{2}+2(P-a) k+\frac{1}{3}(P-2 a)} .
$$

Now, to each of these sums we apply the Jacobi triple product identity (4.1) to get

$$
\begin{aligned}
\sum_{n=0}^{\infty}(-1)^{\lfloor(n+2) / 4\rfloor} q^{a_{n}}=\left(q^{6 P}, q^{3 P+2 a}, q^{3 P-2 a} ; q^{6 P}\right)_{\infty} & \\
& +q^{(P-2 a) / 3}\left(q^{6 P}, q^{5 P-2 a}, q^{P+2 a} ; q^{6 P}\right)_{\infty} .
\end{aligned}
$$

The sum of these two products simplifies to the single product on the right-hand side of (10.8) as is seen by applying (8.2) with $N=2 P$ and $u=q^{(5 P+2 a) / 3}$.

The following corollary covers Theorem 30 .

Corollary 37. Let $P$ be an odd prime power different from a power of 3 , and let a be a positive integer relatively prime to $P$ with $P / 2<a<P$. Let $\left(a_{n}\right)_{n \geq 0}$ be the sequence of non-negative integers $m$ such that $3 P m+a^{2}$ is a square.

(1) If $a \equiv P(\bmod 3)$, then

$$
\sum_{n=0}^{\infty}(-1)^{\lfloor 5 n / 4\rfloor} q^{a_{n}}=\frac{\left(q^{(4 P-4 a) / 3}, q^{(2 P+4 a) / 3}, q^{2 P} ; q^{2 P}\right)_{\infty}}{\left(q^{(5 P-2 a) / 3}, q^{(P+2 a) / 3} ; q^{2 P}\right)_{\infty}}
$$

(2) If $a \not \equiv P(\bmod 3)$, then

$$
\sum_{n=0}^{\infty}(-1)^{\lfloor(n+2) / 4\rfloor} q^{a_{n}}=\frac{\left(q^{(4 P+4 a) / 3}, q^{(2 P-4 a) / 3}, q^{2 P} ; q^{2 P}\right)_{\infty}}{\left(q^{(5 P+2 a) / 3}, q^{(P-2 a) / 3} ; q^{2 P}\right)_{\infty}}
$$

Proof. Here, one goes through the proof of Theorem 36. Everything can be copied, except that the description of the two cases to be considered now reads as shown below, and that this requires a modification of the sign in Case (C1').

$\left(\mathrm{C} 1^{\prime}\right)$ If $a \equiv P(\bmod 3)$, then $S \equiv a, 2 P-a, P+a, 3 P-a(\bmod 3 P)$.

$\left(\mathrm{C} 2{ }^{\prime}\right)$ If $a \not \equiv P(\bmod 3)$, then $S \equiv P-a, a, 3 P-a, 2 P+a(\bmod 3 P)$. 
The following theorem covers Theorems 31 and 32 .

Theorem 38. Let a be 1 or 3 . Furthermore, let $\left(a_{n}\right)_{n \geq 0}$ be the sequence of non-negative integers $m$ such that $16 m+a^{2}$ is a square. Then

$$
\sum_{n=0}^{\infty} q^{a_{n}}=\left(q^{8} ; q^{8}\right)_{\infty}\left(-q^{4+a} ; q^{8}\right)_{\infty}\left(-q^{4-a} ; q^{8}\right)_{\infty}
$$

Proof. We have to find all $S$ such that

$$
S^{2} \equiv a^{2} \quad(\bmod 16),
$$

or, equivalently,

$$
(S-a)(S+a) \equiv 0 \quad(\bmod 16) .
$$

Since $a$ is odd, only one of the factors $N-a$ and $N+a$ can be divisible by 4 . Hence, either $S \equiv a(\bmod 8)$ or $S \equiv-a(\bmod 8)$. Consequently, we have

$$
\begin{aligned}
\sum_{n=0}^{\infty} q^{a_{n}} & =\sum_{k=0}^{\infty} q^{\frac{1}{16}\left((8 k+a)^{2}-a^{2}\right)}+\sum_{k=1}^{\infty} q^{\frac{1}{16}\left((8 k-a)^{2}-a^{2}\right)} \\
& =\sum_{k=0}^{\infty} q^{4 k^{2}+a k}+\sum_{k=1}^{\infty} q^{4 k^{2}-a k}=\sum_{k=-\infty}^{\infty} q^{4 k^{2}+a k}
\end{aligned}
$$

The proof is completed by applying the Jacobi triple product identity (4.1).

\section{Consequences And open problems}

In this section, we record a consequence of Theorem 1 that is inspired by earlier work of Andrews and the second author [1. Furthermore, we end by reminding the reader of a conjecture from [3] related to Theorems 1, 6.

In [1], the function $M_{k}(n)$ is defined as the number of partitions of $n$ in which $k$ is the least positive integer that is not a part and there are more parts $>k$ than there are parts $<k$. For example, if $n=18$ and $k=3$ then we have $M_{3}(18)=3$ because the three partitions in question are

$$
5+5+5+2+1, \quad 6+5+4+2+1, \quad \text { and } 7+4+4+2+1 .
$$

Let $A(n)$ be the number of the partitions of $n$ into parts not congruent to $0,7,8$, $13,15,20,22,27,28(\bmod 35)$ and the parts congruent to 4, 9, 11, 16, 19, 24, 26, 31 (mod 35) have two colours.

We have the following corollary of Theorem 1 .

Corollary 39. Let $k$ and $n$ be positive integers. With $a_{n}$ and $t(n)$ as in Theorem 1 , we have

$$
(-1)^{k-1}\left(\sum_{j=-(k-1)}^{k}(-1)^{j} A(n-j(3 j-1) / 2)-\delta(n)\right)=\sum_{j=0}^{n}(-1)^{t(j)} M_{k}\left(n-a_{j}\right),
$$

where

$$
\delta(n)= \begin{cases}(-1)^{t(m)}, & \text { if } n=a_{m} \\ 0, & \text { otherwise }\end{cases}
$$


Proof. Elementary generating function calculus gives

$$
\sum_{n=0}^{\infty} A(n) q^{n}=\frac{1}{\left(q, q^{4} ; q^{5}\right)_{\infty}\left(q^{2}, q^{3}, q^{4}, q^{5} ; q^{7}\right)_{\infty}} .
$$

Similarly, it is not difficult to see that the generating function for the numbers $M_{k}(n)$ is given by

where

$$
\sum_{n=0}^{\infty} M_{k}(n) q^{n}=\sum_{n=k}^{\infty} \frac{q^{\left(\begin{array}{c}
k \\
(
\end{array}\right)+(k+1) n}}{(q ; q)_{n}}\left[\begin{array}{l}
n-1 \\
k-1
\end{array}\right]
$$

$$
\left[\begin{array}{l}
n \\
k
\end{array}\right]= \begin{cases}\frac{(1-q)\left(1-q^{2}\right) \cdots\left(1-q^{n}\right)}{(1-q)\left(1-q^{2}\right) \cdots\left(1-q^{k}\right)(1-q)\left(1-q^{2}\right) \cdots\left(1-q^{n-k}\right)}, & \text { if } 0 \leqslant k \leqslant n, \\
0, & \text { otherwise, }\end{cases}
$$

is the usual $q$-binomial coefficient.

Andrews and the second author [1] proved the following truncated form of Euler's pentagonal number theorem (1.1):

$$
\frac{(-1)^{k-1}}{(q ; q)_{\infty}} \sum_{n=-(k-1)}^{k}(-1)^{n} q^{n(3 n-1) / 2}=(-1)^{k-1}+\sum_{n=k}^{\infty} \frac{q^{\left(\begin{array}{c}
k \\
2
\end{array}\right)+(k+1) n}}{(q ; q)_{n}}\left[\begin{array}{l}
n-1 \\
k-1
\end{array}\right] .
$$

Multiplying both sides of (11.3) by (11.1), and using Theorem 1 and (11.2), we obtain

$$
\begin{array}{r}
(-1)^{k-1}\left(\left(\sum_{n=1}^{\infty} A(n) q^{n}\right)\left(\sum_{n=-(k-1)}^{k}(-1)^{n} q^{n(3 n-1) / 2}\right)-\sum_{n=0}^{\infty}(-1)^{t(n)} q^{a_{n}}\right) \\
=\left(\sum_{n=0}^{\infty}(-1)^{t(n)} q^{a_{n}}\right)\left(\sum_{n=0}^{\infty} M_{k}(n) q^{n}\right) .
\end{array}
$$

The assertion of the corollary now follows by comparing coefficients of $q^{n}$ on both sides of this equation.

According to (11.3), for $k>0$, the coefficients of $q^{n}$ in the series

$$
(-1)^{k-1}\left(\frac{1}{(q ; q)_{\infty}} \sum_{j=-(k-1)}^{k}(-1)^{j} q^{j(3 j-1) / 2}-1\right)
$$

are all zero for $0 \leqslant n<k(3 k+1) / 2$, and for $n \geqslant k(3 k+1) / 2$ all the coefficients are positive. Related to this result on truncated pentagonal number series, we remark that there is substantial numerical evidence that there is in fact a stronger result.

Conjecture 40. For $k>0$, the coefficients of $q^{n}$ in the series

$$
\begin{aligned}
(-1)^{k-1}\left(\frac{1}{(q ; q)_{\infty}} \sum_{j=-(k-1)}^{k}(-1)^{j} q^{j(3 j-1) / 2}-1\right)\left(q, q^{6}, q^{7} ; q^{7}\right)_{\infty} & \\
= & \frac{(-1)^{k}}{\left(q^{2}, q^{3}, q^{4}, q^{5} ; q^{7}\right)_{\infty}} \sum_{j=k}^{\infty}(-1)^{j} q^{j(3 j+1) / 2}\left(1-q^{2 j+1}\right)
\end{aligned}
$$


are all zero for $0 \leqslant n<k(3 k+1) / 2$ and $n=k(3 k+1) / 2+1$. For $n=k(3 k+1) / 2$ and $n \geqslant k(3 k+1) / 2+2$ all the coefficients are positive.

Remark. The equality above follows easily from (1.1) and little manipulation.

If we assume Conjecture 40, then we immediately deduce that the partition function $A(n)$ satisfies the following infinite families of linear inequalities.

Conjecture 41. For $k>0$, we have

$$
(-1)^{k-1}\left(\sum_{j=-(k-1)}^{k}(-1)^{j} A(n-j(3 j-1) / 2)-\delta(n)\right) \geqslant 0,
$$

with strict inequalities if $n=k(3 k+1) / 2$ or $n \geqslant k(3 k+1) / 2+2$.

To conclude the article, we want to recall a conjecture from [3] that is very similar in appearance to Conjecture 40 and is related (again via (1.1)) to Theorems 16.

Conjecture 42. For $k>0$ and $S \in\{1,2,3,4,5,6\}$, the coefficients of $q^{n}$ in the series

$$
\frac{(-1)^{k}}{\left(q, q^{4} ; q^{5}\right)_{\infty}} \sum_{j=k}^{\infty}(-1)^{j} q^{7 j(j+1) / 2-j S}\left(1-q^{(2 j+1) S}\right)
$$

and

$$
\frac{(-1)^{k}}{\left(q^{2}, q^{3} ; q^{5}\right)_{\infty}} \sum_{j=k}^{\infty}(-1)^{j} q^{7 j(j+1) / 2-j S}\left(1-q^{(2 j+1) S}\right)
$$

are non-negative.

\section{ACKNOWLEDGEMENT}

We thank the anonymous referees for a very careful reading of the original manuscript.

\section{REFERENCES}

[1] G. E. Andrews and M. Merca, The truncated pentagonal number theorem, J. Combin. Theory Ser. A 119 (2012), 1639-1643.

[2] G. Gasper and M. Rahman, Basic Hypergeometric Series, Encyclopedia of Mathematics And Its Applications 35, Cambridge University Press, Cambridge, 1990.

[3] M. Merca, Truncated theta series and Rogers-Ramanujan functions, Exp. Math. (to appear), https://doi.org/10.1080/10586458.2018.1542642.

[4] R. Miranda, Algebraic Curves and Riemann Surfaces, Graduate Studies in Mathematics, vol. 5, Amer. Math. Soc., Providence, R.I., 1995.

[5] T. Miyake, Modular Forms, translated from the Japanese by Y. Maeda, Springer-Verlag, Berlin, 1989.

[6] P. Paule and C.-S. Radu, Partition analysis, modular functions, and computer algebra, in: Recent Trends in Combinatorics, IMA Vol. Math. Appl., vol. 159, Springer, Cham, 2016, pp. 511-543.

[7] E. T. Whittaker and G. N. Watson, A Course of Modern Analysis, reprint of the 4th ed. (1927), Cambridge University Press, Cambridge, 1996.

[8] Y. Yang, Defining equations of modular curves, Adv. Math. 204 (2006), 481-508. 
(C. Krattenthaler) Universität Wien, Fakultät Für Mathematik, Oskar-MorgensternPlatz 1, A-1090 Vienna, Austria. WWW: https://www.mat.univie.ac.at/ kratt.

(M. Merca) Department of Mathematics, University of Craiova, Craiova, Romania; Academy of Romanian Scientists, Bucharest, Romania

(C.-S. Radu) Research Institute for Symbolic Computation, J. Kepler University Linz, A-4040 LinZ, Austria. WWW: https://risc.jku.at/m/cristian-silviu-radu/ 